TRANSACTIONS OF THE

AMERICAN MATHEMATICAL SOCIETY

Volume 363, Number 9, September 2011, Pages 4853-4876

S 0002-9947(2011)05254-2

Article electronically published on April 8, 2011

\title{
SOME RESULTS ON TROPICAL COMPACTIFICATIONS
}

\author{
MARK LUXTON AND ZHENHUA QU
}

\begin{abstract}
In this paper, we establish some further results on tropical compactifications. We give an affirmative answer to a conjecture of Tevelev in characteristic 0: any variety contains a Schön very affine open subvariety. Also we show that any fan supported on the tropicalization of a Schön very affine variety produces a Schön compactification. As an application, we show that the moduli space of six points of $\mathbb{P}^{2}$ in linear general position is Hübsch. Using toric schemes over a discrete valuation ring, we extend tropical compactifications to the nonconstant coefficient case.
\end{abstract}

\section{INTRODUCTION AND STATEMENT OF RESULTS}

Compactification problems play an important role in algebraic geometry. Tropical compactification is introduced and developed by Tevelev in 12] as certain nice compactification for subvarieties of tori. This is applied to the study of compactification of moduli spaces of del Pezzo surfaces in a subsequent paper [3. Our motivation for this paper comes from an attempt to solve the following problem using the idea of tropical compactification.

Conjecture 1.1 ([5]). The Chow quotient compactification $\bar{X}(3, n)$ has log canonical singularity and is the log canonical model of $X(3, n)$ when $n=6,7,8$, where $X(r, n)$ is the moduli space of order $n$ hyperplanes in $\mathrm{P}^{r-1}$ in linear general position.

1.2. We recall some basic terminology of tropical compactification $[12$. Let $Y \subset T$ be a subvariety of an algebraic torus over an algebraically closed field $k$. Let $X(\Delta)$ be a toric variety containing $T$ and let $\bar{Y}$ be the closure of $Y$ in $X(\Delta)$. We say $\bar{Y}$ is a tropical (resp. Schön) compactification if $\bar{Y}$ is proper and the structure map $T \times \bar{Y} \rightarrow X(\Delta)$ is flat (resp. smooth) and surjective.

1.3. We say $Y$ is very affine if $Y$ can be embedded into a torus. The lattice of units $M_{Y}:=\mathcal{O}^{*}(Y) / k^{*}$ is a free abelian group of finite rank. When $Y$ is very affine, $Y$ is embedded into the intrinsic torus Spec $k\left[M_{Y}\right]$ once we choose a section $M_{Y} \rightarrow \mathcal{O}^{*}(Y)$. We say $Y$ is $S c h \ddot{n} n$ if there exists a Schön compactification in the intrinsic torus; if this Schön compactification at the same time is the log canonical model of $Y$, we say that $Y$ is Hübsch. It is conjectured by Tevelev [12] that any variety contains an open very affine Schön subvariety. We show that this is true assuming char $k=0$.

Received by the editors March 22, 2009 and, in revised form, October 11, 2009 and November $18,2009$.

2010 Mathematics Subject Classification. Primary 14E25; Secondary 14 T99.

Key words and phrases. Tropical compactification, Schön variety.

(C)2011 American Mathematical Society 
Theorem 1.4. Assuming char $k=0$, any variety contains an open very affine Schön subvariety.

We show that being Schön is an intrinsic property of $Y$ in the following sense.

Theorem 1.5. If $Y \subset T$ admits a Schön compactification, then any fan $\Delta$ supported on $\operatorname{trop}(Y)$ produces a Schön compactification.

This is very useful in applications. One usually verifies a Schön compactification in a refined fan structure on $\operatorname{trop}(Y)$; then one can claim that it is also true for the minimal fan structure (if it exists). As an application, we will show the following.

Theorem 1.6. $X(3,6)$ is Hübsch.

The rest of the paper is devoted to generalizing tropical compactifications to the nonconstant coefficient case, which is probably more interesting in tropical geometry. In the nonconstant coefficient case we consider a very affine variety $Y$ defined over the field of Puiseux series over $k$; $\operatorname{trop}(Y)$ is then a polyhedral complex which may not admit a fan structure. However $\operatorname{trop}(Y)$ can be realized as the fibre of a map of sets $\mathcal{T}(Y) \rightarrow \mathbb{Q}_{\geq 0}$ at 1 , where $\mathcal{T}(Y)$ is the closure of the cone over $\operatorname{trop}(Y)$ in $N_{\mathbb{Q}} \oplus \mathbb{Q} \cdot \mathcal{T}(Y)$ does admit fan structures and we take it as a replacement of $\operatorname{trop}(Y)$ in the nonconstant coefficient case. This leads us to consider relative toric varieties, or toric schemes over a discrete valuation ring. Using $\mathcal{T}(Y)$ and toric schemes, we are able to generalize tropical, Schön and Hübsch compactifications to the nonconstant coefficient case. With some appropriate modification, Theorems 1.4 and 1.5 remain true in this case.

This paper is organized in the following way. Some preliminaries on tropicalization and tropical compactification are included in section 2. We prove Theorem 1.4 in section 3, Theorem 1.5 in section 4 and Theorem 1.6 in section 5. Section 6 introduces toric schemes over a discrete valuation ring in order to extend our results to the nonconstant coefficient case, which is done in section 7 .

\section{Preliminaries}

We recall the basics of tropical geometry; see for example [2, 10]. Let $k$ be an algebraically closed field of any characteristic, and let $\mathbb{K}$ be the algebraic closure of $k(t)$ with a natural valuation $v: \mathbb{K}^{*} \rightarrow \mathbb{Q}$.

Definition 2.1. For a subscheme $Y \subset T \cong\left(\mathbb{K}^{*}\right)^{n}$, the tropicalization of $Y$ is defined to be

$$
\operatorname{trop}(Y)=\left\{\left(v\left(y_{1}\right), \ldots, v\left(y_{n}\right)\right) \in \mathbb{Q}^{n} \mid\left(y_{1}, \ldots, y_{n}\right) \in Y(\mathbb{K})\right\} \subset \mathbb{Q}^{n} .
$$

It has several equivalent descriptions.

Theorem 2.2 ([10], 2.1.2). The following sets are equal:

(1) $\operatorname{trop}(Y)$ defined as above.

(2) The set $\left\{\left(u\left(t_{1}\right), \ldots, u\left(t_{n}\right)\right) \mid u: \mathcal{O}(Y) \rightarrow \mathbb{Q} \cup\{\infty\}\right\}$, where $u$ runs over all ring valuations $\mathcal{O}(Y) \rightarrow \mathbb{Q} \cup\{\infty\}$ extending $v$.

(3) The set of $w \in \mathbb{Q}^{n}$ such that $\operatorname{in}_{w} f$ is not a monomial for any $f \in I \backslash\{0\}$, or equivalently $\operatorname{in}_{w} Y \neq \emptyset$.

The set in Theorem[2.2(2) is also called a BG-set (short for Bieri-Groves set, [1]). $\operatorname{trop}(Y)$ is a polyhedral complex of pure dimension $(=\operatorname{dim} Y)$. If $I$ is generated by a set of functions in $k\left[t_{1}^{ \pm}, \ldots, t_{n}^{ \pm}\right]$, then we may think of $Y$ as a subscheme of 
$T_{k}$, and we call this the constant coefficient case. When we are in the constant coefficient case, $\operatorname{trop}(Y)$ is conical and admits a fan structure. In this section we consider the constant coefficient case only.

Tropicalization is functorial in the following sense. Given a commutative diagram

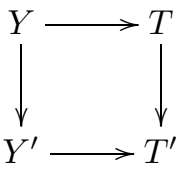

where the horizontal maps are closed embeddings, $T \rightarrow T^{\prime}$ is a homomorphism of tori, then we have an induced commutative diagram

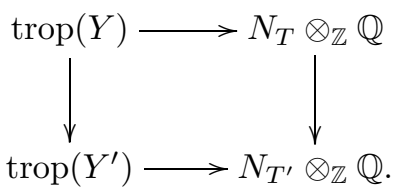

If $Y \rightarrow Y^{\prime}$ is dominant, then $\operatorname{trop}(Y) \rightarrow \operatorname{trop}\left(Y^{\prime}\right)$ is onto.

Now assume that $Y$ is a closed (irreducible) subvariety of $T_{k}$, let $M$ be the lattice of characters of $T$, and $N$ the dual of $M$. The set $\operatorname{trop}(Y)$ can be naturally considered in $N_{\mathbb{Q}}=N \otimes_{\mathbb{Z}} \mathbb{Q}$. Let $\Delta \subset N_{\mathbb{Q}}$ be a fan, denote by $X(\Delta)$ the corresponding toric variety, and $\bar{Y}$ (or $\bar{Y}(\Delta)$ when one wants to specify the fan $\Delta$ ) the closure of $Y$ in $X(\Delta)$. Recall the definitions in Definition 1.2, The basic result about tropical compactification is the following.

Theorem 2.3 (12]). Tropical compactifications exist for any closed subvariety $Y \subset T_{k}$. If $Y(\Delta)$ is a tropical compactification, then $\Delta$ is supported on $\operatorname{trop}(Y)$ and $\bar{Y}\left(\Delta^{\prime}\right)$ is also a tropical compactification, where $\Delta^{\prime}$ is any refinement of $\Delta$. If $Y \subset T$ admits a Schön compactification, then any tropical compactification is Schön.

Remark 2.4. It is often useful to consider an arbitrary closed subscheme $Y \subset T$. The reason is that if $\bar{Y}$ is a tropical (resp. Schön) compactification of an irreducible variety $Y$, and $W \subset X(\Delta)$ is a toric orbit closure, then $\bar{Y} \cap W$ has flat (resp. smooth) surjective structure map in the toric variety $W$, but $\bar{Y} \cap O$ is usually not irreducible where $O \subset W$ is the open orbit. We can define tropical and Schön compactification of an arbitrary closed scheme $Y \subset T$ in the same way. It remains true in this general case that tropical compactification exists, and $|\Delta|=\operatorname{trop}(Y)$ (see for example Proposition (7.4). Indeed, Tevelev's constructive proof works when $Y$ is reduced (not necessarily irreducible). For the general (nonreduced) case, one can use a flattening technique of Raynaud and Gruson [8]. If $Y$ is a closed subscheme and $\bar{Y}$ is a Schön compactification, then $Y$ is necessarily reducible. Since $T \times \bar{Y} \rightarrow X(\Delta)$ is smooth, we conclude that $\bar{Y}$ is a disjoint union of irreducible components. Let $\bar{Y}^{\prime} \subset \bar{Y}$ be an irreducible component. Then there is a possibly smaller toric open subset $X^{\prime} \subset X(\Delta)$ containing $\bar{Y}^{\prime}$ with a smooth surjective structure map. It follows that the fan of $X^{\prime}$, being a subfan of $\Delta$, is supported on $\operatorname{trop}\left(\bar{Y}^{\prime} \cap T\right)$.

Geometric tropicalization computes the set trop $(Y)$ in terms of a nice compactification of $Y$. Let $\bar{Y}$ be a compactification with a simple normal crossing divisorial boundary. For each boundary divisor $D$, the valuation val $_{D}$ restricted on $M_{Y}$ determines a point in $N_{Y}$, still denoted by $\operatorname{val}_{D}$. For each collection $S$ of boundary 
divisors with nonempty intersection, let $\sigma_{S}$ be the cone in $N$ generated by val $_{D}$ with $D \in S$. Then we have the following theorem.

Theorem $2.5([3]) \cdot \operatorname{trop}(Y)$ is the union of all $\sigma_{S}$, where $S$ runs over all collections of boundary divisors with nonempty intersection.

Remark 2.6. The collection of $\sigma_{S}$ does not form a fan in general. In the proof of Theorem 1.4, we search a nice compactification whose geometric tropicalization produces a fan with simplicial cones.

Remarks 2.7. In Theorem 2.5, the requirement for the boundary being a simple normal crossing can be weakened. In fact, when $Y \subset \bar{Y}$ is a toroidal embedding without self-intersection (as defined in [6]), Theorem 2.5 remains true.

Mumford associates to every toroidal embedding $Y \subset \bar{Y}$ a conical polyhedral complex with integral structure $\Delta=\left(|\Delta|, \sigma^{S}, M^{S}\right)$, whose cells are in one-to-one correspondence with the strata of $\bar{Y}$. Recall that for a strata $S, M^{S}$ is the group of Cartier divisors of $\operatorname{Star}(S)$ supported on $\operatorname{Star}(S) \backslash Y, M_{+}^{S}$ is the submonoid of $M^{S}$ consisting of effective Cartier divisors, $N^{S}=\operatorname{Hom}\left(M^{S}, \mathbb{Z}\right)$, and the cell $\sigma^{S}$ of $\Delta$ is spanned by $v \in N^{S}$ such that $\langle v, D\rangle \geq 0$ for any $D \in M_{+}^{S}$.

Assuming $T=\operatorname{Spec} k[M]$, we have a morphism $M \rightarrow M^{S}$ for any stratum $S$, namely $m \mapsto(m)$ on $\operatorname{Star}(S)$, hence a corresponding dual map $N^{S} \rightarrow N$ and $|\Delta| \rightarrow N_{\mathbb{Q}}$. The image of $\sigma^{S} \subset N_{\mathbb{Q}}^{S}$ in $N_{\mathbb{Q}}$ is exactly $\sigma_{S}$; thus the image of $|\Delta|$ is $\bigcup_{S} \sigma_{S}$ as in Theorem 2.5 (here we make no difference between a stratum $S$ and the collection of divisors containing $S$ ).

Let $\Delta^{\prime}$ be a subdivision of $\Delta$ such that all cells are strictly simplicial. By the theory of toroidal embeddings, a subdivision corresponds to another toroidal embedding $Y \subset \bar{Y}^{\prime}$ with a canonical birational morphism $\bar{Y}^{\prime} \rightarrow \bar{Y}$. $\bar{Y}^{\prime}$ has simple normal crossing boundary divisors. By Theorem 2.5, the image of $\left|\Delta^{\prime}\right|$ is $\operatorname{trop}(Y)$, so is the image of $|\Delta|$.

Recall the definitions in 1.3 , Let $Y$ be a very affine variety, and $Y \rightarrow T_{\text {in }}$ be the intrinsic embedding. For any other embedding $Y \rightarrow T$, after a suitable translation, it factors through $T_{\text {in }}$ for a homomorphism of tori $T_{\mathrm{in}} \rightarrow T$. The intrinsic tropicalization has the richest structure and dominates all others.

Definition 2.8. Given $Y \subset T$, we say $Y$ is $S c h o ̈ n$ in $T$ if it admits a Schön compactification for some $X(\Delta) \supset T$. If such a Schön compactification is at the same time the log canonical model of $Y$, then we say $Y$ is Hübsch in $T$.

Theorem 2.9 ([3, 1.10). If $Y \subset T$ is Hübsch, then $\operatorname{trop}(Y)$ has a minimal fan structure $\Delta$, and $\bar{Y}(\Delta)$ is the log canonical compactification.

Theorem 2.10 ([3, 3.1). Let $Y \subset T$ be a Schön closed subvariety. Then either $Y$ is log minimal, or $Y$ is preserved by a nontrivial subtorus of $T$.

Lemma 2.11. If $Y$ is Schön (resp. Hübsch) in some torus $T$, then $Y$ is Schön (resp. Hübsch) in the intrinsic torus.

Proof. Without loss of generality, we can assume $T_{\text {in }} \rightarrow T$ is surjective. Let $\Delta$ be a Schön fan supported on $\operatorname{trop}(Y) \subset N_{T} \otimes_{\mathbb{Z}} \mathbb{Q}$, and let $\Delta^{\prime}$ be any fan supported on the intrinsic tropicalization $\operatorname{trop}(Y) \subset N_{T_{\text {in }}} \otimes_{\mathbb{Z}} \mathbb{Q}$ such that the induced map $\Delta \rightarrow \Delta^{\prime}$ is a fan map. Letting $\bar{Y}$ and $\bar{Y}^{\prime}$ be closures in $X(\Delta)$ and $X\left(\Delta^{\prime}\right)$ respectively, we 
have a fibre diagram

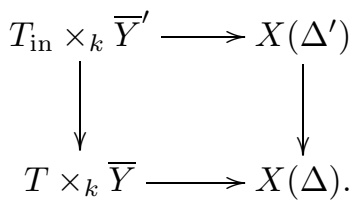

Now suppose $Y \subset T$ is Hübsch. Let $\Delta$ be the canonical (minimal) fan supported on $\operatorname{trop}(Y) \subset N_{\mathbb{Q}}$. The compactification of $Y$ in $X(\Delta)$ is the log canonical model for $Y$, denoted by $\bar{Y}_{\text {lc }}$. Let $\Delta^{\prime}$ be any fan supported on the intrinsic tropicalization and let $\bar{Y}^{\prime}$ be the closure of $Y$ in $X\left(\Delta^{\prime}\right)$; thus we have a canonical birational morphism $\bar{Y}^{\prime} \rightarrow \bar{Y}_{\text {lc }}$. Recall that a ray $\rho$ in $\Delta^{\prime}$ corresponds to some boundary divisors in $\bar{Y}^{\prime}$ (namely $\bar{Y}^{\prime} \cap V_{\rho}$ ) and a collection of rays forms a fan if the corresponding divisors intersect, similarly for $\bar{Y}_{\text {lc }}$; thus the map $\bar{Y}^{\prime} \rightarrow \bar{Y}_{\text {lc }}$ induces a fan map $\Delta^{\prime} \rightarrow \Delta$. We may use $\bar{Y}_{\text {lc }}$ to get a fan structure on the intrinsic $\operatorname{trop}(Y)(\operatorname{Remark} 2.7)$. Note that in Theorem 2.5, these cones do not necessarily form a fan, but here they do if we ignore repeated cones because the images of these cones in $N_{T} \otimes_{\mathbb{Z}} \mathbb{Q}$ form a fan, and 1-dimensional cones map to 1-dimensional cones. Call this fan $\Delta_{\mathrm{lc}}$. Then the closure of $Y$ in $X\left(\Delta_{\mathrm{lc}}\right)$ recovers $\bar{Y}_{\mathrm{lc}}$.

\section{EMBEDDING INTO TORIC VARIETIES}

We prove Theorem 1.4 in this section. Now assume char $k=0$. It suffices to prove the theorem for a smooth variety $Y$. Our strategy is to first compactify $Y$ so that the compactification $\bar{Y}$ is a smooth projective variety and the boundary $\bar{Y} \backslash Y$ is a simple normal crossing divisor. We then study how to embed $\bar{Y}$ into a toric variety $X$ with a one-to-one correspondence between the strata of $\bar{Y}$ and the toric strata of $X$ and with a smooth structure map. Certain requirements have to be imposed on the log structure of $Y$ (Theorem 3.1). These requirements are achieved when we add more generic hyperplane sections to $Y$.

The problem of embedding an arbitrary variety (possibly singular and nonprojective) into some toric variety is studied in 13. Embedding a variety into a given toric variety is equivalent to giving a compatible log structure. Our proof has a similar flavor. We also notice that in [3, the authors obtained some similar requirements using the quotient of affine conoid technique, but only applicable when the Picard group $\operatorname{Pic} \bar{Y}$ is a free abelian group of finite rank.

Let $Y \subset \bar{Y}$ be a (partial) smooth compactification with simple normal crossing divisorial boundary $B=\bigcup_{i \in I} D_{i}$. We have a stratification induced by $B$, where a stratum is defined by $\bigcap_{j \in J} D_{j}-\bigcup_{i \in I \backslash J} D_{i}$ for a subset $J \subset I$; when $J=\emptyset$, the corresponding stratum is the open complement $Y=\bar{Y} \backslash B$. We don't require each stratum to be irreducible. This is a little different from the definition in [6] where a stratum is an irreducible component of our stratum here, but it is more convenient for our purposes for avoiding repeated cones in the geometric tropicalization. For a stratum $S$, let $D_{S}$ be the set $\left\{D_{i} \mid S \subset D_{i}\right\}$, and let $\operatorname{Star}(S)$ be $Y-\bigcup_{D_{i} \notin D_{S}}$. Since $B$ is a simple normal crossing, a stratum $S$ is regular (not necessarily irreducible), and also $\# D_{S}=\operatorname{codim} S$.

Fix $(M, \varphi)$, a pair of an abstract lattice $M$ and a group homomorphism $\varphi: M \rightarrow$ $\mathcal{O}^{*}(Y)$ such that $k[M] \rightarrow \mathcal{O}(Y)$ is a surjection. For any submonoid $M^{\prime} \subset M$, we have an induced $k$-algebra homomorphism $\varphi_{*}: k\left[M^{\prime}\right] \rightarrow \mathcal{O}(Y)$. For any stratum $S$, 
let $\sigma_{S}^{\vee} \subset M$ be the submonoid of $M$ consisting of all $m \in M$ such that $m$ is regular on $\operatorname{Star}(S)$ or equivalently $\operatorname{val}_{D} m \geq 0$ for any $D \in D_{S}$. By abuse of notation sometimes we use $m$ for which we actually mean $\varphi(m)$, for example $\operatorname{val}_{D} m$. Let $N$ be the dual of $M$, and for any stratum $S$, let $\sigma_{S} \subset N_{\mathbb{Q}}$ be the dual cone of $\sigma_{S}^{\vee}$ which is generated by $\operatorname{val}_{D}$ for $D \in D_{S}$. Let

$$
M^{S}:=\left\{m \in M \mid \operatorname{val}_{D} m=0, \forall D \in D_{S}\right\}
$$

i.e., $m$ extends to a unit on $\operatorname{Star}(S)$.

Theorem 3.1. With the notation as above, $Y \subset \bar{Y}$ is a partial compactification. Assume the following conditions are satisfied:

(1) For any stratum $S$, either $\operatorname{Star}(S)$ is affine and the induced map $k\left[\sigma_{S}^{\vee}\right] \rightarrow$ $\mathcal{O}(\operatorname{Star}(S))$ is surjective, or $S$ is affine, and the induced map $k\left[M^{S}\right] \rightarrow \mathcal{O}(S)$ is surjective.

(2) For any stratum $S \neq Y$, and any $D \in D_{S}$, there exists $m \in M$ such that $\mathrm{val}_{D} m=1$ and $\operatorname{val}_{D^{\prime}} m=0$ for any $D^{\prime} \in D_{S} \backslash\{D\}$.

(3) The collection of cones $\left\{\sigma_{S}\right\}$ as $S$ runs over all strata is a fan $\Delta$.

Then there is a canonical closed immersion $Y \rightarrow X(\Delta)$ and $S=Y \cap O_{\sigma_{S}}$ as a scheme-theoretic intersection for any stratum $S$. Hence the structure map is smooth and surjective. When $\bar{Y}$ is proper, $\bar{Y} \subset X(\Delta)$ is a Schön compactification.

Remarks 3.2. If $\operatorname{Star}(S)$ is very affine, then $k\left[\sigma_{S}^{\vee}\right] \rightarrow \mathcal{O}(\operatorname{Star}(S))$ is automatically surjective. If $S$ is very affine, and the restriction map $M^{S} \rightarrow M_{S}$ is surjective, then $k\left[M^{S}\right] \rightarrow \mathcal{O}(S)$ is surjective.

Condition (2) in the above theorem is equivalent to that $\operatorname{val}_{D}$ for $D \in D_{S}$ forms part of a basis of $N$. If $\Lambda$ is the subgroup of $\operatorname{Pic} \bar{Y}$ generated by boundary divisors, then it is also equivalent to that $\Lambda$ is generated by boundary divisors not in $D_{S}$.

Condition (3) in the above theorem is equivalent to the following: for any two strata $S, S^{\prime}$, there exists a unit $m \in M$ such that $\operatorname{val}_{D} m>0$ for all $D \in D_{S} \backslash D_{S^{\prime}}$, $\operatorname{val}_{D} m=0$ for all $D \in D_{S} \cap D_{S^{\prime}}$ and $\operatorname{val}_{D} m<0$ for all $D \in D_{S^{\prime}} \backslash D_{S}$.

Proof of Theorem [3.1. We have a canonical homomorphism of rings $k\left[\sigma_{S}^{\vee}\right] \rightarrow$ $\mathcal{O}(\operatorname{Star}(S))$, and correspondingly $\operatorname{Star}(S) \rightarrow \operatorname{Spec} k\left[\sigma^{\vee}\right]=: U_{S}$. If $S^{\prime}$ is another stratum such that $S^{\prime} \subset \bar{S}$, then we have a commutative diagram

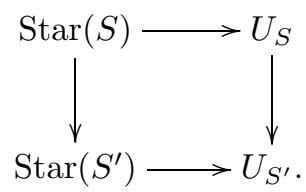

$\sigma_{S}$ is a face of $\sigma S^{\prime}$ and the two vertical arrows are inclusions. By gluing schemes and morphisms, we have a canonical morphism $\bar{Y} \rightarrow X(\Delta)$. This is a closed embedding if and only if for each stratum $S \subset \bar{Y}$, the induced morphism $S \rightarrow X(\Delta)$ is an immersion.

Let $m_{D}^{S} \in M$ be a unit with valuation 1 on $D$ and 0 on other divisors in $D_{S}$. Then $S$ is cut out by $m_{D}^{S}$ on $\operatorname{Star}(S)$ scheme-theoretically. On the other hand $U_{S}$ is isomorphic to $\mathbb{A}^{d} \times \mathbb{G}_{m}^{n-d}$ with $m_{D}^{S}$ as coordinates of $\mathbb{A}^{d}$, where $d=\operatorname{dim} \sigma_{S}$ and $n=\operatorname{rk} M$. Therefore the closed orbit $O_{\sigma_{S}} \subset U_{S}$ is also cut out by $m_{D}^{S}$ schemetheoretically. We conclude that $S$ is the scheme-theoretic intersection $\bar{Y} \cap O_{\sigma_{S}}$. 
If $\operatorname{Star}(S)$ is affine and $k\left[\sigma_{S}^{\vee}\right] \rightarrow \mathcal{O}(\operatorname{Star}(S))$ is surjective, then $\operatorname{Star}(S) \rightarrow U_{S}$ is a closed embedding and $S \rightarrow O_{\sigma_{S}}$ is a closed embedding. If $S$ is affine and $k\left[M^{S}\right] \rightarrow \mathcal{O}(S)$ is surjective, this just says that $S \rightarrow O_{\sigma_{S}}$ is a closed embedding.

The structure map $T_{M} \times Y \rightarrow X(\Delta)$ has fiber $S \times \operatorname{ker}\left(T_{M} \rightarrow O_{\sigma_{S}}\right)$ over any point $x \in O_{\sigma_{S}}$, so it has a reduced, equidimensional, regular fibre. It follows that the structure map is surjective and smooth.

Proof of Theorem 1.4. Let $Y$ be a smooth variety and $\bar{Y}$ be any smooth projective compactification of $Y$ with simple normal crossing boundary divisor $B=$ $\left\{D_{1}, \ldots, D_{r}\right\}$. Let $L$ be a very ample line bundle such that $L+D_{i}$ is very ample for any $i$. Choose a finite set $E_{i} \subset\left|L+D_{i}\right|$ of generic sections with $\# E_{i} \geq$ $\operatorname{dim}\left|L+D_{i}\right|+\operatorname{dim} Y+1$ for $i=0,1, \ldots, r$ where we take $D_{0}$ to be the zero divisor. Let $E$ be the union of all $D_{i}$ and all divisors in $E_{i}, i=0,1, \ldots, r$. By Bertini's theorem, $E$ is a simple normal crossing. Let $Y^{\circ}$ be the complement $\bar{Y} \backslash E$ and $M \cong \mathcal{O}\left(Y^{\circ}\right) / k^{*}$. We show that $(\bar{Y}, E, M)$ satisfies the conditions in Theorem 3.1.

The following facts are frequently used: if $L$ is a very ample line bundle on a projective variety $Y$, and if $s_{1}, \ldots, s_{l}$ are sections of $|L|$ in general linear position with $l=\operatorname{dim}|L|+1$, then the complement is very affine. The intersection of two very affine open subvarieties of $Y$ is again very affine; hence it is also true for $l \geq \operatorname{dim}|L|+1$.

Letting $S$ be any stratum, we have $\# D_{S} \leq \operatorname{dim} Y$. For any $D_{i} \in B \backslash D_{S}$, we pair it with a divisor $D_{i}^{\prime} \in E_{0} \backslash D_{S}$. We can write

$$
E \backslash D_{S}=\bigcup_{i=0}^{r} F_{i}
$$

where $F_{i}=E_{i} \backslash D_{S}$ or $\left(E_{i} \backslash D_{S}\right) \cup\left\{D_{i}+D_{i}^{\prime}\right\}$ if $D_{i} \in D \backslash D_{S}$. All $F_{i} \subset\left|L+D_{i}\right|$ are sections in general linear position with $\# F_{i} \geq \operatorname{dim}\left|L+D_{i}\right|+1$; $\operatorname{thus} \operatorname{Star}(S)=$ $\bigcap\left(\bar{Y} \backslash F_{i}\right)$ is very affine.

Let $D \in D_{S}$ be any divisor. If $D=D_{i} \in B$, we choose some $D^{\prime} \in F_{0}$ and consider $D+D^{\prime} \in\left|L+D_{i}\right|$; otherwise $D \in E_{i} \subset\left|L+D_{i}\right|$ for some $i$. We can find another divisor $G \in E_{i} \backslash D_{S}$, such that $G \neq D$ or $D+D^{\prime}$. There is a unit $m \in M$ with the associated divisor $(m)=D-G$ or $D+D^{\prime}-G$. In either case, this $m$ satisfies condition (2) of Theorem 3.1

To verify condition (3), let $S, S^{\prime}$ be any two strata. For each $D \in D_{S} \backslash D_{S^{\prime}}$, as in the above argument, we can find $m_{D} \in M$ such that $\left(m_{D}\right)=D-G$ (or $D+D^{\prime}-G$ if $D \in B$, for some $\left.D^{\prime} \in E_{0} \backslash\left(D_{S} \cup D_{S^{\prime}}\right)\right)$ and $G \notin D_{S}$. Let $m$ be the product of all $m_{D}$. Then $m$ has positive valuation on $D \in D_{S} \backslash D_{S^{\prime}}, 0$ valuation on $D \in D_{S} \cap D_{S^{\prime}}$ and nonpositive valuation on $D \in D_{S^{\prime}} \backslash D_{S}$. Since $\sigma_{S} \cap \sigma_{S^{\prime}}$ is their common face, condition (3) is satisfied. Similarly we can find $m^{\prime}$ such that $m^{\prime}$ has positive valuation on $D_{S^{\prime}}, 0$ on $D_{S^{\prime}} \cap D_{S}$ and nonpositive valuation on $D_{S} \backslash D_{S^{\prime}}$. Then $m / m^{\prime}$ satisfies condition (3) in Theorem 3.1 for $S, S^{\prime}$.

\section{INTRINSIC PROPERTY OF SCHÖN VARIETIES}

We will prove Theorem 1.5. Let $Y \subset T$ be Schön, and let $\Delta^{\prime}$ be any fan supported on $\operatorname{trop}(Y)$. Let $\Delta$ be a refinement of $\Delta^{\prime}$ such that $\Delta$ is Schön. Denote $\bar{Y}$ and $\bar{Y}^{\prime}$ to be the closures of $Y$ in $X(\Delta)$ and $X\left(\Delta^{\prime}\right)$ respectively. We have a commutative 
diagram,

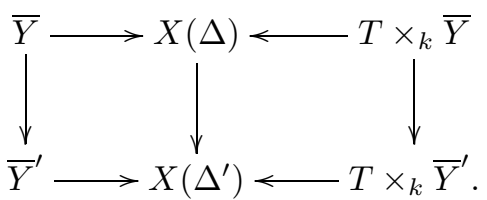

The first step is to show that the left square is a set-theoretic Cartesian diagram, and so is the right square (Theorem 4.4).

Lemma 4.1. Let $Z \subset T$ be a Zariski closed subset of equidimension. If $\operatorname{trop}(Z)$ is preserved by translation by a linear subspace $L$ of $N_{\mathbb{Q}}$, then each irreducible component of $Z$ is preserved by the corresponding subtorus of $T$.

Proof. Let $T \rightarrow T^{\prime}$ be a homomorphism of tori corresponding to $N_{\mathbb{Q}} \rightarrow N_{\mathbb{Q}} / L$. Let $Z_{i} \subset Z$ be irreducible components, and let $Y_{i}$ be the closure of the image of $Z_{i}$ in $T^{\prime}$. Since $\operatorname{trop}\left(Y_{i}\right)$ is the image of $\operatorname{trop}\left(Z_{i}\right)$ in $N_{\mathbb{Q}} / L$, we have $\operatorname{dim} \operatorname{trop}\left(Y_{i}\right) \leq$ $\operatorname{dim} \operatorname{trop}(Z)-\operatorname{dim} L=\operatorname{dim} Z-\operatorname{dim} L$. On the other hand, $\operatorname{dim} \operatorname{trop}\left(Y_{i}\right)=\operatorname{dim} Y_{i} \geq$ $\operatorname{dim} Z_{i}-\operatorname{dim} L$; hence $\operatorname{dim} Z_{i}=\operatorname{dim} Y_{i}+\operatorname{dim} L$. Thus the generic fibre of $Z_{i} \rightarrow Y_{i}$ is the relative torus; hence $Z_{i} \cong Y_{i} \times \operatorname{ker}\left(T \rightarrow T^{\prime}\right)$.

Lemma 4.2. Let $\bar{Y} \subset X(\Delta)$ be a tropical compactification, $W \subset X(\Delta)$ an orbit closure, and $Z=\bar{Y} \cap W$. Then $Z$ is equidimensional and each irreducible component intersects the open orbit in $W$.

Proof. By pulling back the structure map, we see that the structure map of $Z$ in $W$ is also flat. By the openness of flat morphisms, each irreducible component of $Z$ intersects the open orbit $O$ of $W$. Note that $(Z \cap O) \times \operatorname{ker}(T \rightarrow O)$ is the fibre of $T \times \bar{Y} \rightarrow X(\Delta)$ at any point in $O$; thus $Z \cap O$ is equidimensional and so is $Z$.

Lemma 4.3. With the notation and assumptions as in Lemma 4.2, if $p: X(\Delta) \rightarrow$ $X\left(\Delta^{\prime}\right)$ is a proper toric map, then $Z$ is preserved by $T_{W}$, where $T_{W}$ is the relative torus of $W \rightarrow p(W)$.

Proof. Since $Z \subset W$ is a tropical compactification of $Z \cap O, \operatorname{trop}(Z \cap O)$ is the support of the fan of $W$ (see Remark 2.4), which is the inverse image of the support of the fan of $p(W)$ since $p$ is proper. Thus $\operatorname{trop}(Z)$ is preserved by translation of a linear subspace of $N_{O}$ whose corresponding subtorus of $O$ is the relative torus $T_{W}$. The proof then follows from Lemma 4.1.

Theorem 4.4. Let $Y \subset T$ be a closed subvariety, $\bar{Y} \subset X(\Delta)$ a tropical compactification, $p: X(\Delta) \rightarrow X\left(\Delta^{\prime}\right)$ a proper toric map. Then we have a commutative diagram

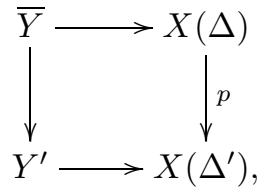

where $Y^{\prime}=p(\bar{Y})$, and then $\bar{Y}$ is a set-theoretic inverse image of $Y^{\prime}$ under the map $X(\Delta) \rightarrow X\left(\Delta^{\prime}\right)$. This applies in particular to the case when $\Delta$ is a refinement of $\Delta^{\prime}$. 
Proof. We show that if $y \in p^{-1}\left(y^{\prime}\right) \cap \bar{Y}$, then $p^{-1}\left(y^{\prime}\right) \subset \bar{Y}$. Letting $O^{\prime}$ be the torus orbit containing $y^{\prime}, p^{-1}\left(O^{\prime}\right)$ is a union of toric varieties with connected fibres. It suffices to show that if $y^{\prime}$ falls in one irreducible component of $p^{-1}\left(O^{\prime}\right)$, then the whole fibre in that irreducible component is contained in $\bar{Y}$. Then this follows from Lemma 4.3 .

Corollary 4.5. With the notation as in Theorem 4.4, let $P$ be the fiber product $Y^{\prime} \times_{X\left(\Delta^{\prime}\right)} X(\Delta)$. Then the induced map $\bar{Y} \rightarrow P$ is the reduction of $P$.

Proof. $P$ is a closed subscheme of $X$ and so is $Y$. The induced map $\bar{Y} \rightarrow P$ is surjective by Theorem 4.4 and since $\bar{Y}$ is integral, we have $\bar{Y}=P_{\text {red }}$.

The following lemma completes the proof of Theorem 1.5 .

Lemma 4.6. In the following commutative diagram, $X, X^{\prime}, Y$ and $Y^{\prime}$ are varieties with $X, X^{\prime}$ and $Y$ normal, and $p$ is proper with connected fibres:

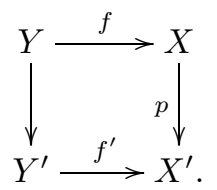

Letting $P=Y \times_{X^{\prime}} X$, assume the induced map $Y \rightarrow P$ is the reduction of $P$. If $f$ is smooth, then so is $f^{\prime}$.

Proof. Letting $y^{\prime} \in Y^{\prime}(k)$ be a closed point, we show that $f^{\prime}$ is smooth at $y^{\prime}$. Let $x^{\prime}=f^{\prime}\left(y^{\prime}\right), F=Y_{x^{\prime}}^{\prime}$ be the scheme-theoretic fibre over $x^{\prime}$ viewed as a closed subscheme of $Y^{\prime}$, and let $G=X_{x^{\prime}}$. Let $\widetilde{Y}$ be the normalization of $Y^{\prime}$. Then the map $Y \rightarrow Y^{\prime}$ factors through $\widetilde{Y}$, as we have the following diagram:

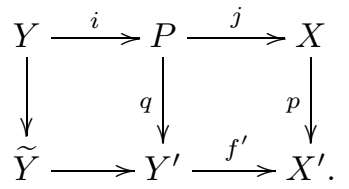

Note that $j^{-1}(G)=F \times_{k} G$, and $f^{-1}(G)$ is a closed subscheme of $j^{-1}(G)$ with the same support, and is smooth over $G$; thus we have $f^{-1}(G)=F_{\text {red }} \times{ }_{k} G$ and $F_{\text {red }}$ is regular.

Let $f_{1}, \ldots, f_{d} \in \mathcal{O}_{F_{\text {red }}, y^{\prime}}$ be a regular system of parameters, and lift them to the local ring $\mathcal{O}_{Y^{\prime}, y^{\prime}}$ with the same notation. Assuming $f_{1}, \ldots, f_{d} \in \Gamma\left(U, \mathcal{O}_{Y^{\prime}}\right)$, we may shrink $U$ a little to assume $U \cap V\left(f_{1}, \ldots, f_{d}\right) \cap F=\left\{y^{\prime}\right\}$.

Let $U \rightarrow X^{\prime} \times{ }_{k} \mathbb{A}_{k}^{d}$ be the map defined by $\left(f^{\prime}, f_{1}, \ldots, f_{d}\right)$, and $\varphi: Y_{U} \rightarrow X \times_{k} \mathbb{A}_{k}^{d}$ be defined by $\left(f, f_{1}, \ldots, f_{d}\right)$. For any point $y \in Y$ lying over $y^{\prime}, f_{1}, \ldots, f_{d}$ restricted in $\mathcal{O}_{f^{-1}(f(y)), y}$ is a regular system of parameters since $f^{-1}(f(y))=F_{\text {red }}$. By Lemma 4.7. $\varphi$ is étale at $y$; thus $\varphi$ is étale in a neighborhood of $Y_{y^{\prime}}$.

Since $p$ has connected fibres, $\widetilde{Y} \rightarrow Y^{\prime}$ is a homeomorphism of the underlying topological spaces; thus there is a unique $\tilde{y} \in \widetilde{Y}$ lying over $y^{\prime}$ and $\varphi$ is also étale in a neighborhood of $Y_{\tilde{y}}$. We have $\varphi^{-1}\left(X_{x}, 0\right)=Y_{y}$, so applying Lemma 4.8 for $W=Y_{y} \subset Y$ and $Z=\left(X_{x}, 0\right) \subset X \times_{k} \mathbb{A}_{k}^{d}$, and the formal function theorem for proper maps $Y \rightarrow \widetilde{Y}$ and $X \times \mathbb{A}^{d} \rightarrow X^{\prime} \times \mathbb{A}^{d}$, we have an isomorphism of formal local rings $\widehat{\mathcal{O}}_{\widetilde{Y}, \tilde{y}} \cong \widehat{\mathcal{O}}_{X^{\prime} \times \mathbb{A}^{d},(x, 0)}$. 
Thus $\tilde{Y} \rightarrow X^{\prime} \times \mathbb{A}^{d}$ is étale at $\tilde{y}$, so it separates tangent vectors at $\tilde{y}$. It follows that $\tilde{Y} \rightarrow Y^{\prime}$ also separates tangent vectors at $\tilde{y}$; hence $\tilde{Y} \rightarrow Y$ is a closed immersion, we have $\widetilde{Y} \cong Y^{\prime}$, and $f^{\prime}: Y^{\prime} \rightarrow X^{\prime}$ is smooth.

Lemma 4.7. Let $f: Y \rightarrow X$ be a morphism of $k$-schemes, $y \in Y(k)$ a closed point. Then $f$ is smooth at $y$ iff there exist $f_{1}, \ldots, f_{d} \in \mathfrak{m}_{y} \subset \mathcal{O}_{Y, y}$ such that the locally defined map $Y \rightarrow X \times{ }_{k} \mathbb{A}_{k}^{d}$ given by $\left(f, f_{1}, \ldots, f_{d}\right)$ is étale at $y$.

Proof. If there is a map $Y \rightarrow X \times{ }_{k} \mathbb{A}_{k}^{d}$ that is étale at $y$, by composing a smooth map $X \times{ }_{k} \mathbb{A}_{k}^{d} \rightarrow X, Y \rightarrow X$ is smooth at $y$. Conversely we know that $Y_{x}$ is regular at $y$. So choose $f_{1}, \ldots, f_{d}$ in $\mathcal{O}_{Y, y}$, which form a regular system of parameters in $\mathcal{O}_{Y_{x}, y}$, and consider the map

$$
\varphi: \mathcal{O}_{X, x}\left[X_{1}, \ldots, X_{d}\right]_{\left(X_{1}, \ldots, X_{d}\right)} \rightarrow \mathcal{O}_{Y, y}
$$

given by $X_{i} \mapsto f_{i}$. $\varphi$ is flat since $\mathcal{O}_{X, x} \rightarrow \mathcal{O}_{X, x}\left[X_{1}, \ldots, X_{d}\right]_{\left(X_{1}, \ldots, X_{d}\right)}$ is faithfully flat, and $\varphi$ is also geometrically regular since the geometric fiber of the closed point is a reduced point; thus $\varphi$ is smooth of relative dimension 0 , hence étale.

Lemma 4.8. Let $f: Y \rightarrow X$ be an étale morphism of schemes, $Z \subset X$ a closed subscheme. If $W=f^{-1} Z \rightarrow Z$ is an isomorphism, then $W_{n} \rightarrow Z_{n}$ is an isomorphism for all $n>0$.

Proof. Let $I$ and $J$ be the ideal sheaves of $Z$ and $W$ respectively. Clearly $J=$ $f^{-1} I \cdot \mathcal{O}_{Y}$, and $J^{n}=f^{-1} I^{n} \cdot \mathcal{O}_{Y}$; thus $W_{n}=f^{-1}\left(Z_{n}\right)$ and we have a morphism $W_{n} \rightarrow Z_{n}$ for each $n>0$ and it is an isomorphism for $n=1$. It follows that $W_{n} \rightarrow Z_{n}$ is a homeomorphism for the underlying spaces and is étale. $Z$ is defined by a nilpotent ideal in $Z_{n}$, by the formal property of étale,

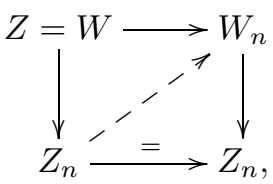

$W_{n} \rightarrow Z_{n}$ admits a section, and then it follows that this is an isomorphism.

The following theorem is an immediate result. A fan $\Delta$ is called convexly disjoint if any convex set contained in $|\Delta|$ is contained in some cone of $\Delta$. If $\Delta$ is convexly disjoint, it is clearly the minimal fan structure on the support. Moreover, it has the following property: for any $\sigma \in \Delta, \operatorname{Star}(\sigma)$ is not preserved by any translation.

Theorem 4.9. Let $Y$ be a Schön very affine variety. If $\bar{Y}\left(\Delta^{\prime}\right) \subset X\left(\Delta^{\prime}\right)$ is a Schön compactification such that $\bar{Y} \cap O_{\sigma^{\prime}}$ is connected for all $\sigma \in \Delta^{\prime}$ except maximal cones, and there exists a convexly disjoint fan $\Delta$ supported on $\operatorname{trop}(Y)$, then $Y$ is Hübsch.

Proof. By Theorem 1.5, $\bar{Y}(\Delta)$ is also a Schön compactification, and we have a fiber diagram

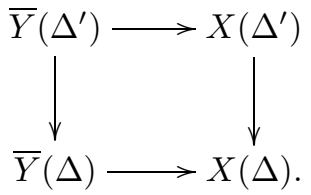

Since $X\left(\Delta^{\prime}\right) \rightarrow X(\Delta)$ has connected fibres, we conclude that $\bar{Y} \cap O_{\sigma}$ is connected for all $\sigma \in \Delta$ except maximal cones. Thus $\bar{Y} \cap O_{\sigma}$ is irreducible and log minimal 
by the fact that $\operatorname{Star}(\sigma)$ is not preserved by a translation and Theorem 2.10. By [3], Theorem 9.1, $\bar{Y}(\Delta)$ is the log canonical model.

\section{The log Canonical model of the moduli space of SiX POINTS IN THE PROJECTIVE PLANE IN GENERAL POSITION}

We regard $X=X(3,6)$ as the space of six general position points in $\mathbb{P}^{2}$ and note that $X$ carries a copy of $M_{0,6}$, the subvariety consisting of arrangements for which all points lie on a conic. Away from $M_{0,6}$, blowing up the six points determines a degree 6 del Pezzo surface $S$ together with a canonical marking of its $(-1)$-curves induced by the blow-up model of $S$. In particular, the complement $Y=Y^{6}=X \backslash M_{0,6}$ may be identified with the moduli space of marked, smooth del Pezzo surfaces of degree 6 , and the root system $E_{6}$ may then be realized as

$$
\left\{\alpha \mid K_{S} \perp \alpha, \alpha^{2}=-2\right\} \subset \operatorname{Pic} S
$$

with positive roots

$$
E_{6}^{+}=\{\beta\} \cup\left\{\alpha_{i j} \mid i<j\right\} \cup\left\{\alpha_{i j k} \mid i<j<k\right\},
$$

where $\beta=2 h-\sum e_{i}, \alpha_{i j}=e_{i}-e_{j}$, and $\alpha_{i j k}=h-e_{i}-e_{j}-e_{k}$. Here, $h$ is the class of a line in $\mathbb{P}^{2}$ and $e_{i}$ is the class of an exceptional divisor. We will often write $I$ for a root of the form $\alpha_{I}$.

$W\left(E_{6}\right)$ acts on $Y$ and in these given coordinates on $E_{6}, Y \subset X$ is $S_{6} \subset W\left(E_{6}\right)$ equivariant. We will make significant use of Naruki's compactification $\bar{Y}$ of $Y$ [] and results in 3 . We recall the following results.

5.1. $Y$ is Hübsch and $\bar{Y}$ is the $\log$ canonical model of $Y . \bar{Y}$ is smooth with simple normal crossing boundary. The stratification $Y \subset \bar{Y}$ satisfies conditions (1), (2) and (3) in Proposition 3.1 ([7, Theorem 1, 3], 1.11, 1.16, 7.4, 8.7, 9.18).

5.2. There are 76 irreducible boundary divisors which form two $W\left(E_{6}\right)$-orbits. The first orbit is in bijective correspondence with the subsystems $\mathcal{A}_{1} \subset E_{6}$, and these are isomorphic to $\bar{M}_{0,6}$. The others are in bijective correspondence with the subsystems $\mathcal{A}_{2} \times \mathcal{A}_{2} \times \mathcal{A}_{2}$, and these are isomorphic to $\bar{M}_{0,4} \times \bar{M}_{0,4} \times \bar{M}_{0,4}$. A collection of divisors corresponding to the subsystems $\Theta_{1}, \ldots, \Theta_{k}$ meet iff, for each pair $\Theta_{i}, \Theta_{j}$, $\Theta_{i} \perp \Theta_{j}$ or one is contained in the other (3 1.14$)$.

5.3. For $\Delta=D_{n}$ or $E_{6}, E_{7}, E_{8}$, let

$$
M(\Delta):=\left\{\sum_{\alpha \in \Delta^{+}} n_{\alpha} \alpha \in \mathbb{Z}^{\Delta^{+}} \mid \sum_{\alpha \in \Delta^{+}} n_{\alpha} \alpha^{2}=0\right\} \subset \mathbb{Z}^{\Delta^{+}} .
$$

$M(\Delta)$ is identified with $M_{Y(\Delta)}$. Under this identification, if $D$ is a boundary divisor of $\bar{Y}(\Delta)$ corresponding to a root subsystem $\Theta \subset \Delta$, then $\operatorname{val}_{D}$ and $\psi(\Theta):=$ $\sum_{\alpha \in \Theta \cap \Delta^{+}} \psi(\alpha)$ are on the same ray, where $\psi: \mathbb{Z}^{\Delta^{+}} \rightarrow N(\Delta)$ is the dual of the inclusion $M(\Delta) \rightarrow \mathbb{Z}^{\Delta^{+}}$. In our case $\Delta=E_{6}, Y=Y^{6}=Y\left(E_{6}\right)$ and $\bar{Y}=\bar{Y}^{6}=$ $\bar{Y}\left(E_{6}\right)$. When $D$ corresponds to an $\mathcal{A}_{1}$ (resp. $\mathcal{A}_{2}^{\times 3}$ ) subsystem, then $\operatorname{val}_{D}=\psi(\Theta)$ (resp. $3 \mathrm{val}_{D}=\psi(\Theta)$ ). See [3], sections 5, 6 and Theorem 7.4.

5.4. We use $\Delta_{Y}$ to denote the minimal fan on $\operatorname{trop}(Y)$. The rays of $\Delta_{Y}$ correspond to subsystems of $E_{6}$ and a collection of rays spans a cone if and only if the corresponding divisors meet. In what follows, we identify a root system with its positive roots and will often use the same notation to refer to a root system, the associated 
ray of $\Delta_{Y}$ and the corresponding divisor. We will need an explicit description of $\Delta_{Y}$. The maximal cones of $\Delta_{Y}$ are spanned by the following rays:

$$
\begin{gathered}
\beta,(i j),(k l),(m n) \\
(i j),(k l),(i j m),(i j n) \\
(i j k),(k l m),(m n),(j l n) \\
\beta,(i j),(k l),(i j m) \amalg(k l n) \\
(i j)(k l)(m n),(i j),(k l),(m n) \\
(i j)(k l)(m n),(i j),(k l),(l k m) \\
(i j)(k l)(m n),(i j),(i j k),(l k m) \\
(i j k) \amalg(l m n),(i j k),(i j),(l m) \\
(i j)(k l)(m n),(i j k),(k l m),(i m n)
\end{gathered}
$$

where

$$
(i j k) \amalg(l m n)=\left\{\beta, \alpha_{i j k}, \alpha_{l m n}\right\} \times\left\{\alpha_{i j}, \alpha_{i k}, \alpha_{j k}\right\} \times\left\{\alpha_{l m}, \alpha_{l n}, \alpha_{m n}\right\}
$$

and

$$
(i j)(k l)(m n)=\left\{\alpha_{i j}, \alpha_{i m n}, \alpha_{j m n}\right\} \times\left\{\alpha_{k l}, \alpha_{i j k}, \alpha_{i j l}\right\} \times\left\{\alpha_{m n}, \alpha_{k l m}, \alpha_{k l n}\right\} .
$$

Note that $(i j)(k l)(m n)=(k l)(m n)(i j)=(m n)(i j)(k l)$. The description of $\Delta_{Y}$ is calculated explicitly in [7].

5.5. Let $D_{4} \subset E_{6}$ be a subsystem. Then there is a canonical homomorphism $N\left(E_{6}\right) \rightarrow N\left(D_{4}\right)$, which corresponds to a KSBA cross ratio of type I, $Y\left(E_{6}\right) \rightarrow M_{0,4}$ under the identification $N\left(E_{6}\right)=N_{Y}$ and $N\left(D_{4}\right)=N_{M_{0,4}}$. The map $N_{Y}=$ $N\left(E_{6}\right) \rightarrow \bigoplus_{D_{4} \subset E_{6}} N\left(D_{4}\right)$ is an injection. The log canonical fan $\Delta_{Y}$ is the intersection fan $\left(N_{Y}\right)_{\mathbb{Q}} \cap \bigoplus \Delta_{4}$, where $\Delta_{4}$ denotes the log canonical fan of $Y\left(D_{4}\right)=M_{0,4}$, which consists of 3 rays in a 2-dimensional space such that the first lattice points add up to 0 . There is a unique decomposition $D_{4}^{+}=F_{1} \amalg F_{2} \amalg F_{3}$ into three quadruples of pairwise orthogonal positive roots, and $M\left(D_{4}\right) \subset \mathbb{Z}^{D_{4}^{+}}$is identified with functions with the same values on elements in one quadruple, and these values add up to $0 . N\left(D_{4}\right)$ consists of 3 rays and each corresponds to an $F_{i}$ (see [3], 5.7, $6.7,9.14)$.

5.6. We will also need a list of $D_{4} \subset E_{6}$. There are two types.

(1) $D(a b c d \mid e)=\{a b, c d, a b e, e d e\} \amalg\{a c, b d, a c e, b d e\} \amalg\{a d, b c, a d e, b c e\}$ (30 of these).

(2) $D(a b|c d| e f)=\{a b, c d, e f, \beta\} \amalg\{a c e, a d f, b c f, b d e\} \amalg\{a c f, a d e, b c d, b d f\}(15$ of these).

Let $U_{\beta}$ be the toric variety determined by the ray $\beta$ and let $\bar{Y}_{\beta}$ be the closure of $Y$ in $U_{\beta}$.

Lemma 5.7. $\bar{Y}_{\beta}$ is isomorphic to $X$.

Proof. Let $D$ be the boundary divisor $X \backslash Y$, since $X$ is very affine, by Proposition 3.1. it suffices to show that val $D=\phi(\beta)$ in $N_{Y}$, which is identified with $N(\Delta)$. This can be done by an explicit calculation.

Note that if $m=\sum_{\alpha \in \Delta^{+}} n_{\alpha} \alpha \in M(\Delta)$ and $\Theta \subset \Delta$ is a subsystem, then $\langle\phi(\Theta), m\rangle=\sum_{\alpha \in \Theta \cap \Delta^{+}} n_{\alpha}$. Recall that the identification $M(\Delta)=M_{Y}$ is obtained as follows: $\mathcal{X}(\Delta)=\left\{x \in \Lambda_{k}^{*} \mid\langle\alpha, x\rangle \neq 0, \forall \alpha \in \Delta^{+}\right\} . M_{\mathcal{X}(\Delta)}$, the units of $\mathcal{X}(\Delta)$, is 
naturally identified with $\mathbb{Z}^{\Delta^{+}}$. Letting $q_{i}=h-3 e_{i}$ be a basis of $\Lambda_{k}$ and $q_{i}^{*}$ the dual basis, define $\phi: \mathcal{X}(\Delta) \rightarrow Y$ to be

$$
x=\sum_{i=1}^{6} a_{i} q_{i}^{*} \mapsto\left(\mathbb{P}^{2},\left[a_{1}: a_{1}^{3}: 1\right], \ldots,\left[a_{6}: a_{6}^{3}: 1\right]\right) .
$$

Then we have an inclusion $M_{Y} \subset M_{\mathcal{X}(\Delta)}=\mathbb{Z}^{\Delta^{+}}$which identifies $M_{Y}$ with $M(\Delta) \subset$ $\mathbb{Z}^{\Delta}([3,6.6)$.

After an automorphism of $\mathbb{P}^{2}$, we may assume, for $\left(\mathbb{P}^{2}, P_{1}, \ldots, P_{6}\right) \in Y, P_{1}=[1$ : $0: 0], P_{2}=[0: 1: 0], P_{3}=[0: 0: 1], P_{4}=[1: 1: 1], P_{5}=[x: y: 1], P_{6}=[z: w: 1]$. Thus we may identify $Y$ with an open subset of $\mathbb{A}^{4}$, removing the divisors $f_{i j k}=0$ and $f_{c}=0$, where $f_{c}=0$ is the divisor corresponding to the six points lying on a conic and $f_{i j k}=0$ is the divisor corresponding to the collinear points $P_{i}, P_{j}, P_{k}$, $1 \leq i<j<k \leq 6$ (excluding $1 \leq i<j<k \leq 4$ and 125, 126). We have $\operatorname{val}_{D} f_{i j k}=0$ and $\operatorname{val}_{D} f_{c}=1$.

$\phi^{*}\left(f_{i j k}\right)=\left(a_{i}-a_{j}\right)\left(a_{j}-a_{k}\right)\left(a_{k}-a_{i}\right)\left(a_{i}+a_{j}+a_{k}\right)=\alpha_{i j}+\alpha_{j k}+\alpha_{i k}+\alpha_{i j k}$. We have $\left\langle\beta, f_{i j k}\right\rangle=0$ and

$$
\begin{aligned}
\phi^{*}\left(f_{c}\right) & =\operatorname{det}\left(a_{i}^{j}\right)_{1 \leq i \leq 6, j=0,1,2,3,4,6} \\
& =-\left(a_{1}+a_{2}+\cdots+a_{6}\right) \prod_{1 \leq i<j \leq 6}\left(a_{i}-a_{j}\right)=\beta+\sum_{1 \leq i<j \leq 6} \alpha_{i j} .
\end{aligned}
$$

We have $\left\langle\beta, f_{c}\right\rangle=1$. This verifies the lemma.

We now regard $\bar{Y}$ as a compactification of $X$ with simple normal crossing boundary divisor. Since $Y \rightarrow X$ is dominant, $\operatorname{trop}(X)$ is the image of $\left|\Delta_{Y}\right|$.

Proposition 5.8. The image of the fan $\Delta_{Y}$ in $N_{X}=N_{Y} / \beta \mathbb{Z}$ is a fan, denoted by $\Delta^{\prime}$, which is strictly simplicial.

Proof. First note that if $D_{4} \subset E_{6}$ is a subsystem, then the image of $\beta$ is 0 in $N\left(E_{6}\right) \rightarrow N\left(D_{4}\right)$ if and only if $\beta \notin D_{4}$. Thus we have a commutative diagram

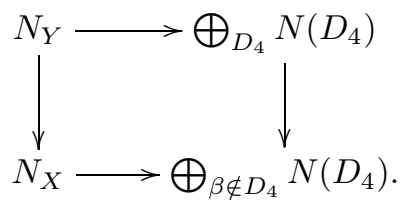

The top arrow is injective. We claim that the bottom arrow is also injective, or equivalently $\bigoplus_{\beta \notin D_{4}} M\left(D_{4}\right) \rightarrow M_{X}$ is surjective. The possible missing units are the units in $M\left(D_{4}\right)$ for $\beta \in D_{4}^{+}=F_{1} \amalg F_{2} \amalg F_{3}$. If $\beta \in F_{1}$, then we may lose the unit $m$ with value 1 on $F_{2}$ and -1 on $F_{3}$. By symmetry, assume $\beta \in D_{4}=D(12|34| 56)$. We note that

$$
\{(135)+(146)+(236)+(245)\}-\{(136)+(145)+(235)+(246)\}
$$

can be written as

$$
\begin{gathered}
(\{(13)+(24)+(135)+(245)\}-\{(14)+(23)+(145)+(235)\}) \\
+(\{(14)+(23)+(146)+(236)\}-\{(13)+(24)+(136)+(246)\}),
\end{gathered}
$$

a sum of a unit in $M(D(1234 \mid 5))$ and a unit in $M(D(1234 \mid 6))$. The claim follows.

To show that the image of $\Delta_{Y}$ is a fan, it is enough to show that if two distinct points $x, y \in\left|\Delta_{Y}\right|$ map to the same point in $\left(N_{X}\right)_{\mathbb{Q}}$, then $x, y$ are in some cone 
$\sigma \in \Delta_{Y}$ such that $\beta$ is a ray of $\sigma$. Assume $y=x+c \beta$ for some $c>0$. Since $\Delta_{Y}$ is the intersection fan $\left(N_{Y}\right)_{\mathbb{Q}} \cap \bigoplus_{D_{4} \subset E_{6}} \Delta_{4}$, the image of $y$ in $\bigoplus \Delta_{4}$ must be in a cone of the form $\bigoplus_{D_{4} \subset E_{6}} \rho\left(D_{4}\right)$, where $\rho\left(D_{4}\right)$ is a ray in $\Delta_{4}$ such that for $\beta \in D_{4}$, $\rho\left(D_{4}\right)$ is the ray containing the image of $\beta$. Thus the image of $x$ is in the same cone. Intersecting $\left(N_{Y}\right)_{\mathbb{Q}}, x, y$ are in the same cone containing $\beta$.

5.9. We again use $(i j),(i j k),(i j k) \amalg(l m n),(i j)(k l)(m n)$ for the image of these in $N_{X}$. We verify the following two relations by a direct computation in every projection $N_{X} \rightarrow N(D(a b c d \mid e))$.

(1) $(i j k) \amalg(l m n)=(i j k)+(l m n)$ in $N_{X}$.

(2) $(i j)(k l)(m n)+(i j)(k l)(m n)=(i j)+(k l)+(m n)$.

Recall that for an $A_{2}^{\times 3}$ type subsystem $\Theta \subset E_{6}, \psi(\Theta)$ is divisible by 3. Here $(i j k) \amalg(l m n)$ and $(i j)(k l)(m n)$ mean the valuation lattice points of the corresponding divisors, which are $\frac{1}{3} \psi((i j k) \amalg(l m n))$ and $\frac{1}{3} \psi((i j)(k l)(m n))$. By symmetry, for case (1), one can only check for the maps $N_{X} \rightarrow N(D(i j k l \mid m))$ and $N_{X} \rightarrow N(D(i j l m \mid k))$. For case (2), one can only check $N_{X} \rightarrow N(D(i j k l \mid m))$ and $N_{X} \rightarrow N(D(i j k m \mid l))$. The computation is left to the reader. We see that the image of the cones

$$
(i j k) \amalg(l m n),(i j k),(i j),(l m) \text { and }(i j k) \amalg(l m n),(l m n),(i j),(l m)
$$

is the single cone spanned by $(i j k),(l m n),(i j),(l m)$, and the two cones spanned by

$$
(i j)(k l)(m n),(i j),(k l),(m n) \text { and }(i j)(m n)(k l),(i j),(k l),(m n)
$$

span a single cone

$$
(i j)(k l)(m n),(i j)(m n)(k l),(i j),(k l),(m n),
$$

which we call a bipyramid cone. Let $\Delta_{X}$ be the fan with the above combined cones and other cones in $\Delta^{\prime}$.

Remark 5.10. Note that $(i j k) \amalg(l m n)$ is no longer a ray in $\Delta_{X}$ (10 of these). By counting numbers, we see that $\Delta_{X}$ has $65=76-10-1$ rays, 990 simplicial maximal cones and 15 bipyramid cones. We recovered the description of the tropicalization of $G(3,6)$ in [11] by a geometric method.

Theorem 5.11. $\Delta_{X}$ is the intersection fan $\left(N_{X}\right)_{\mathbb{Q}} \cap \bigoplus_{\beta \notin D_{4}} \Delta_{4}$. In particular $\Delta_{X}$ is convexly disjoint. With the exception of the 15 bipyramid cones, $\Delta_{X}$ is strictly simplicial.

Proof. Let $\sigma \in \Delta_{X}$ be a maximal cone. We consider the following 3 cases.

(1) $\sigma$ is not a combined cone, and the inverse image in $\Delta_{Y}$ is a maximal cone $\sigma^{\prime}$ which maps isomorphically onto $\sigma$.

(2) $\sigma$ is spanned by $(i j k),(l m n),(i j),(l m)$.

(3) $\sigma$ is spanned by $(i j)(k l)(m n),(i j)(m n)(k l),(i j),(k l),(m n)$.

For case $(1), \sigma^{\prime}=\left(N_{Y}\right)_{\mathbb{Q}} \cap \tau^{\prime}$ for some cone $\tau \in \bigoplus_{D_{4}} \Delta_{4}$; thus $\sigma=\left(N_{X}\right)_{\mathbb{Q}} \cap \tau$, where $\tau$ is the image cone of $\tau^{\prime}$.

For cases (2) and (3), we first check that $\sigma \rightarrow \Delta_{4}$ is a fan map for each $D_{4} \subset E_{6}$ not containing $\beta$. For case (3), by symmetry one can only check for $D(i j k l \mid m)$ and $D(i j k m \mid l)$. For case $(2)$, recall that $D(a b c d \mid e)=F_{1} \amalg F_{2} \amalg F_{3}$ if $(i j) \in F_{1}$. Then for any other three, it is either in $F_{1}$ or not in $D(a b c d \mid e)$ and similarly for $(l m)$. If both $(i j),(l m)$ are not in $D(a b c d \mid e)$, by symmetry, one can only check for $D(i k \ln \mid j)$. The computation is left to the reader. 
Let $\sigma\left(D_{4}\right)$ be the image cone of $\sigma$ in $N\left(D_{4}\right)$ and let $\sigma^{\prime}$ the intersection cone $\left(N_{X}\right)_{\mathbb{Q}} \cap \bigoplus_{\beta \notin D_{4}} \sigma\left(D_{4}\right)$; clearly $\sigma \subset \sigma^{\prime}$. To show that $\sigma=\sigma^{\prime}$, it is enough to show that for a subset $\mathcal{D} \subset\left\{D_{4} \subset E_{6} \mid \beta \notin D_{4}\right\}$, the induced map $\sigma \subset \sigma^{\prime} \rightarrow \bigoplus_{D_{4} \in \mathcal{D}} \sigma\left(D_{4}\right)$ is an isomorphism of $\sigma$ onto the image of $\sigma^{\prime}$. Note that the image of $\sigma^{\prime}$ is the intersection of the image of $\left(N_{X}\right)_{\mathbb{Q}}$ with the cone $\bigoplus_{D_{4} \in \mathcal{D}} \sigma\left(D_{4}\right)$ in $\bigoplus_{D_{4} \in \mathcal{D}} N\left(D_{4}\right)_{\mathbb{Q}}$.

For case $(2)$, we take $\mathcal{D}=\{D(i k \ln \mid j), D(i k \ln \mid m), D(i j k l \mid m), D(\operatorname{lm} n i \mid j)\}$ and identify $\bigoplus_{D_{4} \in \mathcal{D}} \sigma\left(D_{4}\right)$ with $\mathbb{Q}_{+}^{4}$. We see that the image of $(i j k)$, (lmn), (ij) and $(l m)$ is the standard basis of $\mathbb{Q}^{4}$; hence $\sigma$ maps isomorphically onto the image of $\sigma^{\prime}$ (which is $\mathbb{Q}_{+}^{4}$ ).

For case $(3)$, we take $\mathcal{D}=\{D(i j k m \mid l), D(i j k m \mid n), D(k \lim \mid n), D(k \lim \mid j)$, $D(m n k i \mid j), D(m n k i \mid l)\}$, and identify $\bigoplus_{D_{4} \in \mathcal{D}} \sigma\left(D_{4}\right)$ with $\mathbb{Q}_{+}^{6}$. We have

$$
\begin{gathered}
(i j)(k l)(m n) \mapsto(1,0,1,0,1,0), \\
(i j)(m n)(k l) \mapsto(0,1,0,1,0,1), \\
(i j) \mapsto(1,1,0,0,0,0), \\
(k l) \mapsto(0,0,1,1,0,0), \\
(m n) \mapsto(0,0,0,0,1,1) .
\end{gathered}
$$

One can use $\psi\left(A_{2}^{\perp}\right)=2 \psi\left(A_{2}\right)$ to simplify the computation ([3], 5.10). The image of $\sigma^{\prime}$ is the intersection of $\mathbb{Q}_{+}^{6}$ with the 4-dimensional subspace given by $x_{1}+x_{4}=$ $x_{2}+x_{3}$ and $x_{1}+x_{6}=x_{2}+x_{5}$. One verifies that this is exactly the cone spanned by the above 5 vectors by checking the intersection with each 3-dimensional face of $\mathbb{Q}_{+}^{6}$ given by $x_{i}=x_{j}=x_{k}=0$.

We showed that $\Delta_{X}$ is indeed the intersection fan; the other results are immediate.

Proposition 5.12. The stratification induced by $X \subset \bar{Y}$ satisfies condition (1) in Theorem 3.1 .

Proof. The stratification on $\bar{Y}$ induced by $X \subset \bar{Y}$ (resp. $Y \subset \bar{Y}$ ) is denoted by $\mathcal{S}$ (resp. $\mathcal{S}^{\prime}$ ). Let $S$ be a stratum in $\mathcal{S}$; we may assume $\operatorname{dim} S>0$. There is a unique stratum $S^{\circ}$ in $\mathcal{S}^{\prime}$ such that $S^{\circ} \subset S$ is an open subset. There are two cases to consider:

(1) $S^{\circ}=S$; in this case $\beta$ and $\sigma_{S}$ do not span a cone in $\Delta_{Y}$.

(2) $S^{\circ} \subsetneq S$; in this case $S^{\circ}$ is the complement of the divisor of $S$ cut out by $\beta$. We shall show that $S$ is very affine and that $M_{X}^{S} \rightarrow M_{S}$ is surjective; i.e. $M_{S}$ is generated by the restriction of units on $X$ with 0 valuation on $D \in D_{S}$ (or equivalently units on $Y$ with 0 valuation on $D \in D_{S}$ and $\beta$ ).

For case (1), it is clear that $S$ is very affine since $S$ is also a stratum in $\mathcal{S}^{\prime}$. We first assume that $\sigma_{S}$ is a ray. Then $\sigma_{S}$ is of the form $(i j k)$ or $(i j)(k l)(m n)$, and $S$ is isomorphic to $M_{0,6}$ or $M_{0,4}^{\times 3}$. For $\sigma_{S}=(i j k)$, a morphism $X \rightarrow Y\left(D_{4}\right)$ for some $D_{4}$ not containing $\beta$ extends to a unit on the stratum $S$ if and only if $(i j k) \notin D_{4}$. Thus we have a commutative diagram

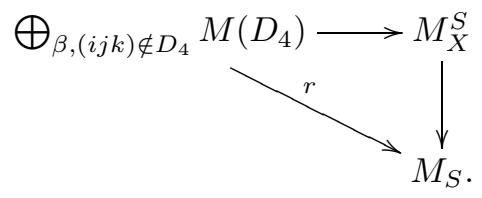

To show that $r$ is a surjection, we make a $W\left(E_{6}\right)$ action which sends $(i j k)$ to $\beta$ and $\beta$ to some $\left(i^{\prime} j^{\prime} k^{\prime}\right)$. Then $S$ is sent to $D=X \backslash Y$, which is identified with the locus 
where 6 points lie on a conic. We need to show that $\bigoplus_{\beta,\left(i^{\prime} j^{\prime} k^{\prime}\right) \notin D_{4}} M\left(D_{4}\right) \rightarrow M_{X}^{D}$ is a surjection. A $D_{4}$ cross ratio of the form $D(a b c d \mid e)$ corresponds to the morphism $X \rightarrow M_{0,4}$ defined by the four points $a, b, c, d$ on the conic passing through $a, b, c, d, e$. This is explicit in the proof of [3], Theorem 6.6. Thus the extension map restricted on $D=M_{0,6}$ is forgetting $e$ and $f$, where $f$ is the 6 th point. It is well known that these units generate the units on $M_{0,6}$. There are $30 D_{4} \subset E_{6}$ not containing $\beta$ (of the form $D(a b c d \mid e)$ ). They form 15 pairs with same forgetful map $(D(a b c d \mid e)$ and $D(a b c d \mid f)$ form a pair). At least one in each pair does not contain $\left(i^{\prime} j^{\prime} k^{\prime}\right)$, which implies the result.

For $\sigma_{S}=(i j)(k l)(m n)=\Theta$, a cross ratio of the form $D_{4}=D(a b c d \mid e)=$ $F_{1} \amalg F_{2} \amalg F_{3}$ extends to a unit on $S$ if and only if $\langle\psi(\Theta), m\rangle=0$ for any $m \in M\left(D_{4}\right)$, or equivalently $\left|\Theta \cap F_{1}\right|=\left|\Theta \cap F_{2}\right|=\left|\Theta \cap F_{3}\right|$. Among $D($ abcd $\mid e)$, those of the form $D(i j k m \mid n)$ will do (12 of these). The 9 divisors of $\bar{M}_{0,4}^{\times 3}$ are intersections with divisors $\{(i j),(i m n),(j m n)\},\{(k l),(k i j),(l i j)\}$ and $\{(m n),(m k l),(n k l)\}$. The divisors in the same group are disjoint. It's easy to check that the units of $M_{0,4}^{\times 3}$ induced from the first component (cut out by the divisors $(i j),(i m n),(j m n)$ ) are the restrictions of the cross ratio map corresponding to $D(i j k m \mid n)$ and similarly for the other two components.

For $\operatorname{dim} \sigma_{S}>1$, one of the rays of $\sigma_{S}$ is of the form $\rho=(i j k)$ or $(i j)(k l)(m n)$. Letting $Z$ be the corresponding stratum, we view $S$ as a stratum of the closure of $Z$, which is a Schön compactification (either $\bar{M}_{0,6}$ or $\bar{M}_{0,4}^{\times 3}$ with the usual stratification). Thus $S$ is embedded in the corresponding torus orbit of rank rk $M_{Z}-d+1$. We have a commutative diagram

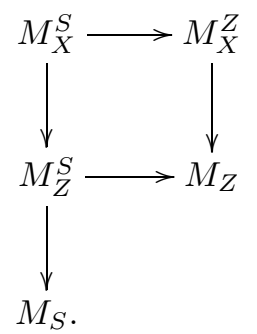

The horizontal arrows are inclusions, $M_{X}^{Z} \rightarrow M_{Z}$ and $M_{Z}^{S} \rightarrow S$ are surjective. $M_{X}^{S} \rightarrow M_{Z}^{S}$ is also surjective by rank counting. We have completed case (1).

For case (2), we have a commutative diagram

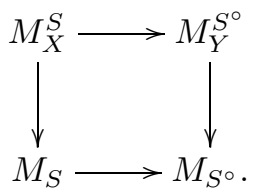

Assume $S$ is very affine, the existence of a unit on $Y$ with valuation 1 on $\beta$ and 0 on $D \in D_{S}$ implies that $\operatorname{rk} M_{S}=\operatorname{rk} M_{S^{\circ}}-1$. Since $\operatorname{rk} M_{X}^{S}=\operatorname{rk} M_{Y}^{S^{\circ}}-1$, and $M_{Y}^{S^{\circ}} \rightarrow M_{S^{\circ}}$ is surjective, so is $M_{X}^{S} \rightarrow M_{S}$.

Finally we need to show that $S$ is very affine. First assume $\operatorname{codim} S=1$. Then $\sigma_{S}$ is either $(i j)$ or $(i j k) \amalg(l m n)$. If $\sigma_{S}=(i j)$, then $S \subset \bar{M}_{0,6}$, removing all boundary divisors except the divisor corresponding to $\beta$, which is a divisor of type $\bar{M}_{0,5}$. If we identify this divisor with $n$ pointed stable rational curves of type $\{1,2,3,4\} \amalg\{5,6\}$, 
and identify the first 3 points with $0, \infty, 1$ after an automorphism of $\mathbb{P}^{1}$, then $S$ is a subset of $\mathbb{A}^{3}$ with coordinates $x_{4}, x_{5}, x_{6}$ satisfying $x_{4}, x_{5}, x_{6} \neq 0,1$ and $x_{4} \neq x_{5}, x_{6}$ (the equation $x_{5}=x_{6}$ defines the divisor cut out by $\beta$ on $S$ ). It is clear that $S$ is very affine. If $\sigma_{S}=(i j)(k l)(m n)$, then $S \subset \bar{M}_{0,4}^{\times 3}$, removing all boundary divisors except one. $S$ is then isomorphic to $\mathbb{G}_{m} \times M_{0,4} \times M_{0,4}$, which is very affine.

For higher codimension, $S$ is the complement of $\bar{M}_{0,4}^{\times i}$ or $\bar{M}_{0,5}$ with one less boundary divisor of the usual boundary. These are clearly very affine.

Theorem 5.13. $X=X(3,6)$ is Hübsch, and $\Delta_{X}$ is the log canonical fan. The log canonical model $\bar{X}_{\mathrm{lc}}$ is smooth with simple normal crossing boundary divisor outside of 15 isolated singularities which form a single $S_{6}$-orbit. Locally analytically, each singularity is isomorphic to $C\left(\mathbb{P}^{1} \times \mathbb{P}^{2}\right)$, the cone over $\mathbb{P}^{1} \times \mathbb{P}^{2}$. The map $\bar{Y}^{6} \rightarrow \bar{X}_{\mathrm{lc}}$ is obtained by 10 divisorial contractions and 15 small contractions.

Proof. By Proposition 5.8 and 5.12 $X \subset \bar{Y}$ satisfies the assumptions in Theorem 3.1; thus $\bar{Y}$ is a Schön compactification of $X$ in $X\left(\Delta^{\prime}\right) . \bar{Y} \cap O_{\sigma^{\prime}}$ is connected for any $\sigma^{\prime} \in \Delta^{\prime}$ by the explicit description of boundary divisors of $\bar{Y}$. By Theorem 5.11 . $\Delta_{X}$, supported on $\operatorname{trop}(X)$, is convexly disjoint. By Theorem 4.9, we conclude that $X$ is Hübsch. Other statements are clear from the description of $\Delta_{X}$.

\section{TORIC SCHEMES OVER A DISCRETE VALUATION RING}

In order to extend tropical compactification to the nonconstant coefficient case, we need to introduce the notion of toric schemes over a discrete valuation ring. This has first appeared in Mumford [6] in an effort of extending the semi-stable reduction theorem. Smirnov [9] constructed toric schemes from certain polyhedra (his so-called admissible polyhedra). We observe that a simple construction is just by pulling back a toric map $X(\Delta) \rightarrow \mathbb{A}_{k}^{1}$ to the spectrum of the local ring at 0 . We shall also compare a toric scheme (with the same combinatorial data) in various ring extensions since we ultimately intend to study a subvariety $Y \subset T$ over the field of Puiseux series.

First let's fix some notation: $(R, \mathfrak{m}, k)$ is a discrete valuation ring with $\mathfrak{m}$ the maximal ideal, $k$ the residue field, and $K$ the quotient field; assume $k \subset R \subset K$. Let $\mathbb{K}$ be the algebraic closure of $K$. We write $\operatorname{Spec} R=\{\eta, s\}$, where $\eta$ is the generic point and $s$ the closed point. For any scheme $X$ over $\operatorname{Spec} R$, we use $X_{\eta}$ and $X_{s}$ to denote the generic fibre and special fibre respectively. We fix $t \in \mathfrak{m} \backslash \mathfrak{m}^{2}$, a uniformizer. Our main application would be that $k$ is an algebraically closed field of characteristic $0, \mathbb{K}$ is the algebraic closure of $k(t)$, i.e. the field of Puiseux series over $k$, and $K=k\left(\left(t^{1 / n}\right)\right)$ for some $n>0$.

Definition 6.1. A (normal) toric scheme over $R$ is an integral normal scheme $\mathfrak{X}$ together with a map $\mathfrak{X} \rightarrow \operatorname{Spec} R$ which is separated and of finite type, such that it contains the torus $T_{K}$ in its generic fibre $\mathfrak{X}_{\eta}$ and there is a group scheme action $T_{R} \times_{R} \mathfrak{X} \rightarrow \mathfrak{X}$ which extends the left multiplication of $T_{K}$ on itself.

To construct a toric scheme, we need the following data. Let $N$ be a lattice of $\operatorname{rank} n, \widetilde{N}=N \oplus \mathbb{Z}, \Delta \subset \widetilde{N}_{\mathbb{Q}}$ a fan such that $\operatorname{pr}_{2}(\Delta) \subseteq \mathbb{Q}_{\geq 0}$. We call such a fan an admissible fan and cones in it admissible cones. This gives a toric morphism of ordinary toric varieties $X(\Delta) \rightarrow \mathbb{A}_{k}^{1}$, the toric scheme $\mathfrak{X}(\Delta) \rightarrow$ Spec $R$ is obtained from $X(\Delta)$ via the base change $\operatorname{Spec} R \rightarrow \mathbb{A}_{k}^{1}$, where the map $\operatorname{Spec} R \rightarrow \mathbb{A}_{k}^{1}$ is 
defined by the map $k[x] \rightarrow R: x \mapsto t$. If $\Delta$ consists of a single cone $\sigma$ together with all its faces, we write $\mathfrak{X}_{\sigma}$ instead of $\mathfrak{X}(\Delta)$.

This construction is essentially the same as in [6]. We shall describe $\mathcal{O}\left(\mathfrak{X}_{\sigma}\right)$ explicitly for $\sigma \in \Delta$. Let $M$ and $\widetilde{M}=M \oplus \mathbb{Z}$ be the dual of $N$ and $\widetilde{N}$ respectively. For a lattice point $\widetilde{m} \in \widetilde{M}$, we write $\widetilde{m}=(m, s)$ where $m \in M$ and $s \in \mathbb{Z}$, and let $e=(0,1) \in \widetilde{M}$. Note that for any admissible cone $\sigma, e$ is contained in its dual cone $\sigma^{\vee}$. If we write $S_{\sigma}$ for the monoid $\sigma^{\vee} \cap \widetilde{M}$, then

$$
\mathcal{O}\left(\mathfrak{X}_{\sigma}\right)=k\left[S_{\sigma}\right] \otimes_{k[x]} R=R\left[\chi^{\widetilde{m}}\right]_{\tilde{m} \in S_{\sigma}} /\left(\chi^{e}-t\right)=R\left[\chi^{m} t^{s}\right]_{(m, s) \in S_{\sigma}} .
$$

Denote $R\left[\chi^{m} t^{s}\right]_{(m, s) \in S_{\sigma}}$ by $A\left[S_{\sigma}\right]$, and note that $A[S]$ can be defined for any submonoid $S \subset \widetilde{M}$ containing $e$. In [6], $\mathfrak{X}(\Delta)$ is constructed by gluing all Spec $A\left[S_{\sigma}\right]$; we refer the reader to [6] IV $\S 3$ for basic properties of $\mathfrak{X}(\Delta)$ which are analogous to ordinary toric varieties; for example the one-to-one correspondence between toric orbits and cones, the correspondence of $T$-linearized invertible sheaves and piecewise linear maps $|\Delta| \rightarrow \mathbb{Q}$, etc. We give a brief proof of the following properties of $\mathfrak{X}(\Delta)$ since they are not included in [6] and are sort of special for toric $R$-schemes.

Proposition 6.2. $\quad$ (1) The generic fiber of $\mathfrak{X}$ is $X(\Delta \cap N)$, where $\Delta \cap N$ is the subfan of $\Delta$ consisting of all cones of $\Delta$ which are in $N \subset \widetilde{N}$.

(2) For any $\rho \in \Delta(1)$, a 1-dimensional cone, let $v_{\rho}$ be the first lattice point on $\rho$. Then $\operatorname{val}_{V_{\rho}}\left(\chi^{m} t^{s}\right)=\left\langle\widetilde{m}, v_{\rho}\right\rangle$, where $\widetilde{m}=(m, s) \in \widetilde{M}$ and $V_{\rho}$ is the closure of the orbit $O_{\rho}$ corresponding to $\rho$.

(3) The special fibre of $\mathfrak{X}(\Delta)$ is a union of irreducible components indexed by $\rho \in \Delta(1)$ not contained in $N$, whose reduction is the union of $V_{\rho}$. The irreducible component indexed by $\rho$ is reduced if and only if $\operatorname{pr}_{2}\left(v_{\rho}\right)=1$, and the special fibre $\mathfrak{X}_{s}$ is reduced if and only if $\operatorname{pr}_{2}\left(v_{\rho}\right)=1$ for all $\rho \not \subset N$.

(4) For an admissible fan $\Delta \subset \widetilde{N}=N \oplus \mathbb{Z}$, let $\Delta[d]$ be the same fan but considered in $N \oplus d \mathbb{Z}$. There exists an integer $d>0$ such that for any discrete valuation ring $R^{\prime}, \mathfrak{X}(\Delta[d])$ as a toric $R^{\prime}$-scheme has reduced special fibre, and furthermore when $R^{\prime}=R\left[t^{1 / d}\right], \mathfrak{X}(\Delta[d])$ is obtained from $\mathfrak{X}(\Delta)$ via the base change Spec $R\left[t^{1 / d}\right] \rightarrow$ Spec $R$ followed by normalization.

Proof. (1) follows easily from the pullback construction of $\mathfrak{X}$ and the toric orbit structure of the ordinary toric variety $X(\Delta)$.

Letting $O_{\sigma}^{\prime}$ and $V_{\sigma}^{\prime}$ be the usual toric orbit and orbit closure of $X(\Delta)$, it is easy to check that $O_{\sigma}$ and $V_{\sigma}$ are obtained via the base change $\mathfrak{X} \rightarrow X(\Delta)$. For (2), note that the rational function $\chi^{m} t^{s} \in K[M]$ on $\mathfrak{X}$ is induced from the rational function $\chi^{\widetilde{m}} \in k[\widetilde{M}]$ on $X(\Delta)$; thus $\operatorname{val}_{V_{\rho}} \chi^{m} t^{s}=\operatorname{val}_{V_{\rho}} \chi^{\widetilde{m}}=\left\langle\widetilde{m}, v_{\rho}\right\rangle$.

For (3), we already know that $\left(\mathfrak{X}_{s}\right)_{\text {red }}=\bigcup_{\rho} V_{\rho}$ for $\rho \not \subset N$. Let $\mathfrak{X}_{s, \rho}$ be the irreducible component indexed by $\rho$, with $V_{\rho}$ its reduction. $\mathfrak{X}_{s, \rho}$ is reduced if and only if $\operatorname{val}_{V_{\sigma}} t=1$, that is, $\operatorname{pr}_{2}\left(v_{\rho}\right)=1$ by $(2)$. This can also be shown by the affine ring. Let $\sigma \in \Delta$; then $\mathcal{O}\left(\mathfrak{X}_{\sigma} \times_{R} k\right)=k\left[\sigma^{\vee} \cap \widetilde{M}\right] /\left(\chi^{e}\right)$, where the effect of modulo $\left(\chi^{e}\right)$ is to make $\chi^{\widetilde{m}}=0$ if $\widetilde{m}-e \in \sigma^{\vee} \cap \widetilde{M}$. If $\operatorname{pr}_{2}\left(v_{\rho}\right)=0$ or 1 , for all $\rho<\sigma$, then $\chi^{\widetilde{m}}=0$ for all $\widetilde{m}$ in the interior of $\sigma^{\vee}$; hence $k\left[\sigma^{\vee} \cap \widetilde{M}\right] /\left(\chi^{e}\right)$ is reduced.

For (4), let $d$ be the least common multiple of $v_{\rho}$ for all $\rho \not \subset N$. Then $\Delta[d]$ satisfies the assumption in (3); hence $\mathfrak{X}(\Delta[d])$ has reduced special fibre. Now assume $R^{\prime}=R\left[t^{1 / d}\right]$. We show that $\mathfrak{X}(\Delta[d])$ is the normalization of $\mathfrak{X}(\Delta) \times_{R} R\left[t^{1 / d}\right]$. This is a local question. We show this for each $\sigma \in \Delta$. Note that the dual of $N \oplus d \mathbb{Z}$ 
is $M \oplus \frac{1}{d} \mathbb{Z}$ and we have a natural embedding $\widetilde{M} \subset M \oplus \frac{1}{d} \mathbb{Z}$ corresponding to $N \oplus d \mathbb{Z} \subset \widetilde{N}$. Let $\sigma[d]$ be the same cone but considered in $N \oplus d \mathbb{Z}$, and $S_{\sigma[d]}$ the monoid $\sigma^{\vee} \cap\left(M \oplus \frac{1}{d} \mathbb{Z}\right)$.

The affine ring of $\mathfrak{X}_{\sigma} \times_{R} R\left[t^{1 / d}\right]$ is $R\left[\chi^{m} t^{s}\right]_{(m, s) \in S_{\sigma}} \otimes_{R} R\left[t^{1 / d}\right]$. The effect of this tensor product is just introducing $\frac{1}{d} e$ in $S_{\sigma}$; i.e., if $S^{\prime}$ is the monoid generated by $S_{\sigma}$ and $\frac{1}{d} e$ in $S_{\sigma[d]}$, then

$$
R\left[\chi^{m} t^{s}\right]_{(m, s) \in S_{\sigma}} \otimes_{R} R\left[t^{1 / d}\right]=R\left[\chi^{m} t^{s}\right]_{(m, s) \in S^{\prime}} .
$$

The effect of normalization is saturating $S^{\prime}$, which is $S_{\sigma[d]}$.

Remark 6.3. If $\mathfrak{X}(\Delta)$ has reduced special fibre, then $\mathfrak{X}(\Delta[d])=\mathfrak{X}(\Delta) \times_{R} R\left[t^{1 / d}\right]$. This is because $S^{\prime}$ (the monoid generated by $S_{\sigma}$ and $\frac{1}{d} e$ in $S_{\sigma[d]}$ ) is already saturated, i.e. $S^{\prime}=S_{\sigma[d]}$.

\section{TROPICAL COMPACTIFICATION IN NONCONSTANT COEFFICIENT CASE}

We first define tropical and Schön compactification for $Y \subset T$ over a field with discrete valuation and establish parallel results as in the constant coefficient case. With the notation as in the previous section, let $Y \subset T_{K}$ be a subvariety. $\operatorname{trop}(Y)$ in this case is taken to be the tropicalization of $Y_{\mathbb{K}} \subset T_{\mathbb{K}}$.

Note that $\operatorname{trop}(Y)$ sits inside $N_{\mathbb{Q}}$ and define $\mathcal{T}(Y)$ to be the closure of the set $\left\{(t \cdot x, t) \in N_{\mathbb{Q}} \oplus \mathbb{Q}=\widetilde{N}_{\mathbb{Q}} \mid x \in \operatorname{trop}(Y), t \in \mathbb{Q}_{>0}\right\}$. We take $\mathcal{T}(Y)$ as a replacement of $\operatorname{trop}(Y)$ in the nonconstant coefficient case. If we modify the BG-set definition of $\operatorname{trop}(Y)$ (Theorem 2.2. (2)), then $\mathcal{T}(Y)$ coincides with the following set:

$$
\left\{\left(\left.u\right|_{M_{\mathbb{Q}}}, u(t)\right) \in N_{\mathbb{Q}} \oplus \mathbb{Q} \mid u: \mathcal{O}(Y) \rightarrow \mathbb{Q}\right\},
$$

where $u$ runs over all valuations trivial on $R^{*}$ and nonnegative on $R$. Letting $\Delta \subset \widetilde{N}_{\mathbb{Q}}$ be an admissible fan, $\mathfrak{X}(\Delta)$ the corresponding toric scheme over $R, \bar{Y}$ the closure of $Y$ in $\mathfrak{X}(\Delta)$, we make the following definitions.

Definition 7.1. We say $\bar{Y}$ is a tropical (resp. Schön) compactification if $\bar{Y}$ is proper over $R$ and the structure map $T_{R} \times_{R} \bar{Y} \rightarrow \mathfrak{X}(\Delta)$ is flat (resp. smooth) and surjective. For a very affine variety $Y$, we say $Y$ is Schön if it admits a Schön compactification in the intrinsic torus.

Definition 7.2. $\bar{Y} \subset \mathfrak{X}(\Delta)$ is called a Hübsch compactification if it is Schön, $\mathfrak{X}(\Delta)$ has reduced special fibre and $K_{\bar{Y}}+B_{\bar{Y}}$ is ample. We say $Y$ is Hübsch in $T$ if it admits a Hübsch compactification in the intrinsic torus.

Remark 7.3. Since $\mathfrak{X}(\Delta)$ has reduced fibre and $\bar{Y}$ has a smooth structure map, $K_{\mathfrak{X}}$ and $K_{\bar{Y}}$ are well defined. We have $K_{\mathfrak{X}}+B_{\mathfrak{X}}=0$, and by the adjunction formula, $K_{\bar{Y}}+B_{\bar{Y}}=\operatorname{det} \mathscr{N}_{\bar{Y} / \mathfrak{X}(\Delta)}$.

Proposition 7.4. $\bar{Y}$ is proper over $R$ if and only if $|\Delta| \supset \mathcal{T}(Y)$. If $\Delta$ is a tropical fan, then $|\Delta|=\mathcal{T}(Y)$.

Proof. This can be proved following the same idea as in the constant coefficient case in 12. Here we proceed with a new proof using the BG-set definition and valuative criterion. 
Suppose $\bar{Y}$ is proper over $R$. Let $u$ be a valuation $K(Z)^{\times} \rightarrow \mathbb{Q}$ which is trivial on $R^{*}$ and nonnegative on $R$ for some subvariety $Z$ of $Y$. Let $R(Z)$ be the valuation ring. Then we have the following commutative diagram:

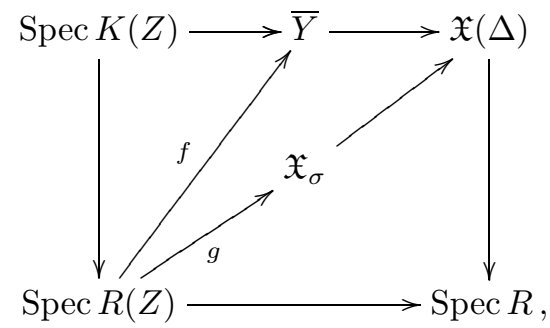

where the existence of $f$ is due to the valuative criterion of properness of $\bar{Y}$. $f$ then factors through some open affine toric scheme $\mathfrak{X}_{\sigma} \subset \mathfrak{X}(\Delta)$. $g$ corresponds to a ring homomorphism $\mathcal{O}\left(\mathfrak{X}_{\sigma}\right) \rightarrow R(Z)$, which implies that $u$ takes nonnegative values on $S_{\sigma}$; thus $[u] \in \sigma$, where $[u]$ denotes the image of $u$ in $\widetilde{N}_{\mathbb{Q}}$. We proved $|\Delta| \supset \mathcal{T}(Y)$.

Suppose $|\Delta| \supset \mathcal{T}(Y)$. If $\bar{Y}$ is not proper, there is a proper $R$-scheme $Y^{\prime}$, containing $\bar{Y}$ as an open dense subscheme. Let $y_{1}$ be the generic point of $Y^{\prime}$ and $y_{0}$ any point of $Y^{\prime}$ not contained in $\bar{Y}$. Then $\mathcal{O}_{Y^{\prime}, y_{0}}$ is dominated by a discrete valuation ring of $K\left(Y^{\prime}\right)=K(Y)$. Let $u$ denote the valuation, and $R(Y)$ the valuation ring. Then $[u] \in \sigma$ for some $\sigma \in \Delta$. The map Spec $R(Y) \rightarrow Y^{\prime}$ sending the generic point to $y_{1}$ and the closed point to $y_{0}$ factors through $\mathfrak{X}_{\sigma}$, a contradiction.

Let $\Delta$ be a tropical fan. We already proved $\Delta \supset \mathcal{T}(Y)$; hence for any $\Delta^{\prime}$ refining $\Delta, \bar{Y}\left(\Delta^{\prime}\right)$ is proper over $R$. Let $\mathfrak{Y}=\bar{Y}(\Delta) \times_{\mathfrak{X}(\Delta)} \mathfrak{X}\left(\Delta^{\prime}\right)$. We show that $\mathfrak{Y}=\bar{Y}\left(\Delta^{\prime}\right)$. Indeed we have a fibre diagram

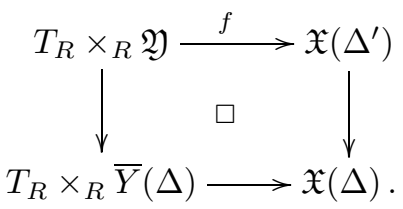

Since $\bar{Y}(\Delta)$ is tropical, the top arrow is also flat and surjective. Restricting on the open subscheme $T_{K} \subset \mathfrak{X}\left(\Delta^{\prime}\right), f^{-1}\left(T_{K}\right)=T_{K} \times_{K} Y$, which is integral. By the lemma below, $T_{R} \times_{R} \mathfrak{Y}$ is integral, so is $\mathfrak{Y}$. Hence $\mathfrak{Y}$ is $\pi^{-1}(\bar{Y}(\Delta)) \subset \mathfrak{X}\left(\Delta^{\prime}\right)$ with reduced induced structure; hence $\bar{Y}\left(\Delta^{\prime}\right)=\mathfrak{Y}$, and it is tropical and is the pullback of $\bar{Y}(\Delta)$.

It remains to prove that $\Delta$ is supported on $\mathcal{T}(Y)$. Suppose it is not. Let $\Delta^{\prime}$ refine $\Delta$ such that there is a subfan $\Delta^{\prime \prime} \subset \Delta^{\prime}$ with $\left|\Delta^{\prime \prime}\right|=\mathcal{T}(Y)$. $\mathfrak{X}\left(\Delta^{\prime \prime}\right)$ is an open subscheme of $\mathfrak{X}\left(\Delta^{\prime}\right)$, but not equal to $\mathfrak{X}\left(\Delta^{\prime}\right) . \bar{Y}\left(\Delta^{\prime \prime}\right)=\bar{Y}\left(\Delta^{\prime}\right)$; thus the structure map $T_{R} \times{ }_{R} \bar{Y}\left(\Delta^{\prime}\right) \rightarrow \mathfrak{X}\left(\Delta^{\prime}\right)$ fails to be surjective.

Lemma 7.5. Let $f: X \rightarrow Y$ be a flat morphism of schemes. Assume $Y$ is integral. If there exists a dense Zariski open subset $U \subset Y$ such that $f^{-1}(U)$ is integral, then $X$ is integral.

Proof. For any $x \in X$, let $y=f(x)$. Take affine open neighborhoods Spec $B$ and $\operatorname{Spec} A$ of $x$ and $y$ respectively such that $f: \operatorname{Spec} B \rightarrow \operatorname{Spec} A$. Then $A \rightarrow B$ is flat. We can find $a \in A$ such that $\operatorname{Spec} A_{a} \subset U$. Since $f^{-1}\left(\operatorname{Spec} A_{a}\right) \cap \operatorname{Spec} B=$ Spec $B_{a}$, we know $B_{a}$ is a domain. Tensoring $0 \rightarrow A \rightarrow A_{a}$ with $B$ over $A$, we have $0 \rightarrow B \rightarrow B_{a}$; hence $B$ is a domain. $X$ is reduced. 
If $X$ is not irreducible, there is an irreducible component $X^{\prime}$ of $X$ such that $f: X^{\prime} \rightarrow Y-U$. However each irreducible component of $X$ should dominate $Y$ by the openness of a flat map. This is a contradiction, which proves the lemma.

Proposition 7.6. Any refinement of a tropical fan is tropical. If $Y \subset T_{K}$ admits a Schön compactification, then any tropical fan produces a Schön compactification. Let $\bar{Y} \subset \mathfrak{X}(\Delta)=\mathfrak{X}$ be a Schön compactification. Then $\bar{Y}$ is locally a complete intersection in $\mathfrak{X}(\Delta)$. Furthermore for any refinement $\Delta^{\prime}$ of $\Delta$, let $\bar{Y}^{\prime}$ be the closure of $Y$ in $\mathfrak{X}\left(\Delta^{\prime}\right)=\mathfrak{X}^{\prime}$ with proper birational map $\pi: \bar{Y}^{\prime} \rightarrow \bar{Y}$. Then $\operatorname{det} \mathscr{N}_{\bar{Y}^{\prime} / \mathfrak{X}^{\prime}}=$ $\pi^{*}\left(\operatorname{det} \mathscr{N}_{\bar{Y} / \mathfrak{X}}\right)$.

Proof. The proof is the same as in the constant coefficient case 12 using the fibre diagram in Proposition 7.4 .

Proposition 7.7. Tropical compactifications exist for any subvariety $Y \subset T_{K}$.

Proof. The proof is the same as in the constant coefficient case using Kapranov's visible contour construction and Laforgue's transversality argument.

Lemma 7.8. Let $Y \subset T_{K}$ be a subvariety and assume $Y$ is geometrically integral. If $\Delta$ produces a tropical (resp. Schön, resp. Hübsch) compactification of $Y$, then $\Delta[d]$ also produces a tropical (resp. Schön, resp. Hübsch) compactification for $Y_{K^{\prime}} \subset T_{K^{\prime}}$, where $K^{\prime}=K\left(t^{1 / d}\right)$.

Proof. Let $R^{\prime}=R\left[t^{1 / d}\right]$ and $\bar{Y}(\Delta[d])$ be the closure of $Y_{K^{\prime}}$ in $\mathfrak{X}(\Delta[d])$. We have a fibre diagram (by Lemma 7.5 )

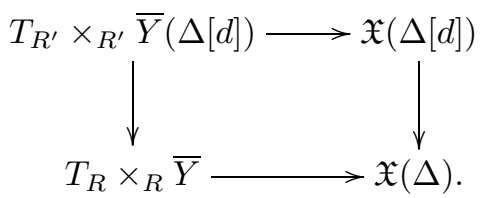

Hence if $\bar{Y}$ is tropical (resp. Schön), so is $\bar{Y}(\Delta[d])$.

If $\bar{Y}$ is Hübsch, $\mathfrak{X}(\Delta)$ has reduced special fibre and so does $\mathfrak{X}(\Delta[d])$. Thus $\bar{Y}(\Delta[d])$ and $\mathfrak{X}(\Delta[d])$ are obtained from $\bar{Y}$ and $\mathfrak{X}(\Delta)$ simply by the ring extension Spec $R^{\prime} \rightarrow \operatorname{Spec} R$ (see Remark 6.3). Therefore $\bar{Y}(\Delta[d])$ is also Hübsch.

Proposition 7.9. If $Y \subset T_{K}$ admits a Hübsch compactification and $Y$ is geometrically integral, then $\mathcal{T}(Y)$ has a minimal fan structure $\Delta$ corresponding to the log canonical compactification.

Proof. Let $\Delta^{\prime}$ be another fan supported on $\mathcal{T}(Y)$. We show that $\Delta^{\prime}$ is a refinement of $\Delta$. Suppose on the contrary that $\Delta^{\prime}$ does not refine $\Delta$. Then there exists a $d$-dimensional cone $\sigma^{\prime} \in \Delta^{\prime}(d=\operatorname{dim} Y)$, which is not contained in any cone of $\Delta$. There is a $(d-1)$-dimensional cone $\alpha \in \Delta$ which meets the interior of $\sigma^{\prime}$. Let $\Delta^{\prime \prime}$ be a common strictly simplicial refinement. Then there is a $(d-1)$-dimensional cone $\alpha^{\prime \prime} \subset \alpha \cap \sigma^{\prime}$, meeting the interior of both $\alpha$ and $\sigma^{\prime}$. We may assume that $\mathfrak{X}(\Delta), \mathfrak{X}\left(\Delta^{\prime}\right)$ and $\mathfrak{X}\left(\Delta^{\prime \prime}\right)$ all have reduced fibre; otherwise consider $\Delta[l], \Delta^{\prime}[l]$ and $\Delta^{\prime \prime}[l]$ for some $l$ (Lemma 7.8).

We have proper birational maps

$$
p_{1}: \mathfrak{X}^{\prime \prime}:=\mathfrak{X}\left(\Delta^{\prime \prime}\right) \rightarrow \mathfrak{X}:=\mathfrak{X}(\Delta)
$$


and

$$
p_{2}: \mathfrak{X}^{\prime \prime} \rightarrow \mathfrak{X}^{\prime}:=\mathfrak{X}\left(\Delta^{\prime}\right) .
$$

Let $Z, Z^{\prime}$ and $Z^{\prime \prime}$ be the orbit closures of $V_{\alpha}, V_{\sigma^{\prime}}$ and $V_{\alpha^{\prime \prime}}$ in $\mathfrak{X}, \mathfrak{X}^{\prime}$ and $\mathfrak{X}^{\prime \prime}$ respectively.

Note that $\sigma^{\prime}$ is not contained in $N$ since it's a maximal cone in $\mathcal{T}(Y)$, and consequently the same holds for $\alpha$ and $\alpha^{\prime \prime}$; hence $Z, Z^{\prime}$ and $Z^{\prime \prime}$ are all on the special fibre, in particular, are all normal toric varieties over $k$.

$Z^{\prime}$ is isomorphic to a torus $T_{k}^{\prime}$. The induced morphism $p_{2}: Z^{\prime \prime} \rightarrow Z^{\prime}$ is a proper toric morphism of relative dimension 1 ; thus $Z^{\prime \prime} \cong Z^{\prime} \times_{k} \mathbb{P}_{k}^{1} . p_{1}: Z^{\prime \prime} \rightarrow Z$ is birational.

Let $\bar{Y}$ and $\bar{Y}^{\prime \prime}$ be the closure of $Y$ in $\mathfrak{X}$ and $\mathfrak{X}^{\prime \prime}$ respectively. $\bar{Y}^{\prime \prime}$ is a Schön compactification with reduced special fibre. The scheme-theoretic intersection $C:=$ $Y^{\prime \prime} \cap Z^{\prime \prime} \subset T_{k}^{\prime} \times_{k} \mathbb{P}_{k}^{1}$ is 1-dimensional, reduced, proper and smooth over $k$. Thus $C \cong z \times \mathbb{P}^{1}$ for some $z \in T_{k}^{\prime}$ a 0 -dimensional reduced closed subscheme. So we have that $K_{C}+B_{C}$ is trivial. Since $\bar{Y}^{\prime \prime} \rightarrow \bar{Y}$ is $\log$ crepant, and $K_{\bar{Y}}+B_{\bar{Y}}$ is ample, by the projection formula we conclude that $C$ is contracted by $p_{1}: Z^{\prime \prime} \rightarrow Z$. Since $p_{1}$ is equivariant, all fibres $z^{\prime} \times \mathbb{P}^{1}$ are contracted, a contradiction to $p_{1}$ being a birational map.

Theorem 7.10. Assume char $K=0$. Then for any variety $Y$ over $K, Y$ contains a Schön very affine variety.

Proof. The proof proceeds in an analogous streamline as in the constant coefficient case, only with some technical issues to be taken care of. Let $Y^{o}$ be a regular very affine variety over $K$ and $\bar{Y}$ a regular compactification, projective over Spec $R$ with reduced special fibre and simple normal crossing boundary divisor. Let $M$ be a lattice with a homomorphism $\phi: M \rightarrow \mathcal{O}^{*}(Y)$ such that $K[M] \rightarrow \mathcal{O}(Y)$ is surjective. For any stratum $S$, let $\widetilde{M}_{S}$ be the set $\{\widetilde{m}=(m, r) \in \widetilde{M}=M \oplus \mathbb{Z} \mid \widetilde{m} \in$ $\mathcal{O}(\operatorname{Star}(S))\}$ (where we think of $\widetilde{m}=\phi(m) t^{r}$ as a rational function on $Y$ ). The conditions in Theorem 3.1 are now the following:

(1) For each stratum $S$, Star $M$ is affine and $A\left[M_{S}\right] \rightarrow \mathcal{O}(\operatorname{Star}(S))$ is surjective.

(2) For each stratum $S$ and any divisor $D_{0} \in D_{S}$, there exists $\widetilde{m} \in \widetilde{M}_{S}$ such that $\operatorname{val}_{D_{0}} \widetilde{m}=1$ and $\operatorname{val}_{D^{\prime}} \widetilde{m}=0$ for all $D^{\prime} \in D_{S} \backslash\left\{D_{0}\right\}$.

(3) The cones $\sigma_{S}$ (the dual cone of $\widetilde{M}_{S}$ ) form an admissible fan in $\widetilde{N}_{\mathbb{Q}}$.

We may assume $Y$ is regular. By Hironaka's resolution theorem and Mumford's semi-stable reduction theorem, there is a compactification $\bar{Y}$, possibly over a ring extension $R \subset R\left[t^{1 / d}\right]$, which is regular with reduced special fibre and simple normal crossing boundary divisor. Thanks to the following lemma of a relative version of Bertini's theorem, the above conditions can be achieved by adding more generic hyperplane sections as in the proof of Theorem 1.4

Lemma 7.11 (4). Let $X$ be a regular scheme, flat and quasi-projective over Spec $R$. Assume that $X_{s}$ is a reduced and simple normal crossing. Then a general hyperplane $H \subset \mathbb{P}_{R}^{n}$ intersects $X$ transversely and $(X \cap H) \cup X_{s}$ is a simple normal crossing.

Theorem 7.12. If $Y$ is Schön in $T_{K}$, then any fan supported on $\mathcal{T}(Y)$ produces a Schön compactification. 
Proof. This is a relative version of Theorem 1.5, which is proved by reducing to the constant case. Let $\Delta^{\prime}$ be any fan supported on $\mathcal{T}(Y)$, and let $\Delta$ be a refinement of $\Delta^{\prime}$ which is Schön. Let $\bar{Y}$ and $\bar{Y}^{\prime}$ be the closures of $Y$ in $\mathfrak{X}(\Delta)$ and $\mathfrak{X}\left(\Delta^{\prime}\right)$ respectively. We have a commutative diagram as follows:

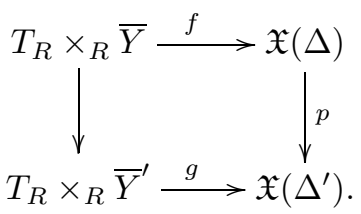

Note that $f$ is smooth. Pulling the diagram back to the generic fibre and lifting to the algebraic closure $\mathbb{K}$, we have

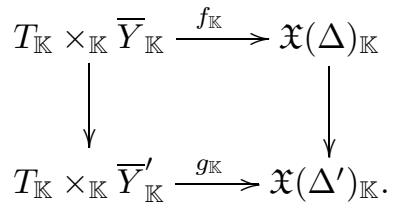

Now $\bar{Y}_{\mathbb{K}}$ is a disjoint union of irreducible components of pure dimension, and each irreducible component has a smooth surjectives structure map possibly in a smaller toric open set (see Remark 2.4). Applying Theorem 4.4 to each irreducible component of $\bar{Y}_{\mathbb{K}}$, we see that $\bar{Y}_{\mathbb{K}}$ is a set-theoretic inverse image of $\bar{Y}_{\mathbb{K}}^{\prime}$; hence $\bar{Y}_{\mathbb{K}}^{\prime}$ is also a disjoint union of irreducible components of pure dimension. We can now apply Theorem 1.5 to each irreducible component of $Y_{\mathbb{K}}$, where $g_{\mathbb{K}}$ is smooth.

Let $W$ be any toric orbit closure of $\mathfrak{X}(\Delta)$ on the special fibre (which is a normal toric variety over $k$ ) and let $O \subset W$ be the open orbit. Then $W \rightarrow p(W)$ is a proper toric map. Restricting the diagram on $W$, we have

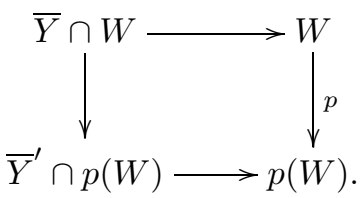

$\bar{Y} \cap W$ has a smooth structure map in $W$. By the same argument as above, we can show that $\bar{Y}^{\prime} \cap p(W)$ also has a smooth structure map. Combining the above result, we see that $f$ is smooth.

Assume that $k$ is of characteristic $0 . \mathbb{K}$ is then the field of Puiseux series over $k$. For a subvariety $Y \subset T_{\mathbb{K}}, Y$ is defined over $K_{n}:=k\left(\left(t^{1 / n}\right)\right)$ for some $n$. We propose the following definition.

Definition 7.13. $Y \subset T_{\mathbb{K}}$ is Schön (resp. Hübsch) if for some $n, Y$ is defined over $K_{n}$ and $Y \subset T_{K_{n}}$ is Schön (resp. Hübsch).

This is to avoid some technical difficulty such as schemes over a non-Noetherian ring. It is clear that if $Y \subset T_{\mathbb{K}}$ is Hübsch, then $\mathcal{T}(Y)$ has a minimal fan structure.

\section{ACKNOWLEDGEMENTS}

We would like to take the opportunity here to thank all those whose conversations and advice were helpful to us, especially our thesis advisor, Séan Keel, for his 
continued support and advice. We also thank E. Katz and J. Tevelev for many helpful discussions.

\section{REFERENCES}

1. R. Bieri and J.R.J. Groves, The geometry of the set of characters induced by valuations, J. Reine Angew. Math. 347 (1984), 168-195. MR0733052 (86c:14001)

2. M. Einseidler, M. Kapranov, and D. Lind, Non-archimedean amoebas and tropical varieties, J. Reine Angew. Math. 601 (2006), 139-157. MR2289207(2007k:14038)

3. P. Hacking, S. Keel, and J. Tevelev, Stable pair, tropical, and log canonical compact moduli of del Pezzo surfaces, Invent. Math. 178 (2009), no. 1, 173-227. MR2534095

4. U. Jannen and S. Saito, Bertini theorems and Lefschetz pencils over discrete valuation rings, with applications to higher class field theory, http://www.mathematik.uniregensburg. de/Jannsen/home/Preprints/Bertini2007-07-01.pdf (preprint) (2007).

5. S. Keel and J. Tevelev, Geometry of Chow quotients of Grassmannians, Duke Math. J. 134 (2006), no. 2, 259-311. MR2248832 (2007m:14017)

6. G. Kempf, F. Knudsen, D. Mumford, and B. Saint-Donat, Toroidal embeddings I, Lecture Notes in Mathematics, no. 339, Springer, 1973. MR0335518 (49:299)

7. I. Naruki, Cross ratio variety as a moduli space of cubic surfaces, Proc. London Math. Soc. 45 (1982), 1-30. MR662660 (84d:14020)

8. M. Raynaud and L. Gruson, Critères de platitude et de projectivité, Invent. Math. 13 (1971), 1-89. MR0308104 (46:7219)

9. A.L. Smirnov, Toric schemes over a discrete valuation ring, St. Petersburg Math. J. 8 (1997), no. 4, 651-659. MR1418258 (98a:14068)

10. D. Speyer, Tropical geometry, Ph.D. thesis, University of California, Berkeley, 2005.

11. D. Speyer and B. Sturmfels, The tropical Grassmannian, Adv. Geom. 4 (2004), 389-411. MR2071813 (2005d:14089)

12. J. Tevelev, Compactifications of subvarieties of tori, Amer. J. of Math. 129 (2007), no. 4, 1087-1104. MR.2343384 (2008f:14068)

13. J. Włodarczyk, Embeddings in toric varieties and prevarieties, J. Algebraic Geom. 2 (1993), 705-726. MR.1227474 (94e:14070)

Department of Mathematics, University of Texas at Austin, Austin, Texas 78712

E-mail address: mluxton@math.utexas.edu

Department of Mathematics, University of Texas at Austin, Austin, Texas 78712

Current address: Department of Mathematics, East China Normal University, Shanghai 200241, People's Republic of China

E-mail address: zhqu@math.ecnu.edu.cn 